\title{
Stability in Yang-Mills Theories
}

\author{
Clifford Henry Taubes* \\ Department of Mathematics, Harvard University, Cambridge, MA 02138, USA
}

\begin{abstract}
At a solution of the Yang-Mills equations on $S^{4}$, or the Yang-MillsHiggs equation on $\mathbb{R}^{3}$, the hessian of the action functional defines a natural second order, elliptic operator. The number of negative eigenvalues of this operator is bounded below by a multiple of the relevant topological charge. The proof of this assertion requires a relative index theorem for Dirac-type operators on $\mathbb{R}^{n}, n \geqq 3$.
\end{abstract}

\section{Introduction}

The Yang-Mills equations on $S^{4}$ and the static Yang-Mills equations on $\mathbb{R}^{4}$ (the Yang-Mills-Higgs equations on $\mathbb{R}^{3}$ ) are both variational equations of functionals on topologically interesting spaces. A solution to the equations is a critical point of the functional. At a critical point, the differential of the functional is zero. The second variation of the functional is called the hessian, and it is a bilinear form on a suitably defined Hilbert space. The index of the critical point is defined to be the number of eigenvectors of the hessian which have negative eigenvalues. The purpose of this article is to prove that because of topological considerations, certain values of the index do not occur for the Yang-Mills functional on $S^{4}$ and the Yang-Mills-Higgs functional on $\mathbb{R}^{3}$.

Consider first the SU(2) Yang-Mills equations on $S^{4}$ (cf. $[1,2]$ for reviews). The function space, $\mathfrak{B}$, is the space of isomorphism classes of pairs $(P, A)$ where $P \rightarrow S^{4}$ is a principal $\mathrm{SU}(2)$-bundle and $A$ is a smooth connection on $P$. With respect to the $C^{\infty}$-topology, $\mathfrak{B}=\bigcup_{n} \mathfrak{B}_{n}$ is the disjoint union of spaces $\mathfrak{B}_{n}$ which are indexed by $n \in \mathbb{Z}$. The integer $n$ is minus the second Chern class of $P \times{ }_{\mathrm{SU}(2)} \mathbb{C}^{2}$. (It is the physicist's instanton number.) Bourguignon et al. [3] have shown that every local minimum of the Yang-Mills functional, $\mathfrak{Y M}$, on $\mathfrak{B}_{n}$ is (anti) self-dual. Nonminimal critical points of $\mathfrak{Y M}$ have yet to be discovered, but if one exists, the following result applies :

* Junior Fellow of the Harvard University Society of Fellows. Supported in part by the NSF under grant No. PHY-82-03669 
Theorem 1.1. Let $P \rightarrow S^{4}$ be a principal SU(2) bundle of degree $n$, and let $A$ be a connection on $P$, and a solution to the Yang-Mills equations. Then the index, $k$, of the hessian of $\mathfrak{Y M}$ at $A$ satisfies $k \geqq 2(|n|+1)$ or else $k=0$ and $A$ is (anti) self-dual.

A theorem like Theorem 1.1 is suggested by the work of Atiyah and Jones [4].

Results for principal $G$-bundles over $S^{4}$ where $G$ is a simple compact Lie group with rank $>1$ are stated in Theorems 2.1 and 2.2.

For the SU(2) Yang-Mills-Higgs equations on $\mathbb{R}^{3}$ (cf. $[5,6]$ ), the relevant function space is

$\mathfrak{B}=\{$ Isomorphism classes of smooth pairs $(A, \Phi)$, where $A$ [a connection on $\left.\mathbb{R}^{3} \times \mathrm{SU}(2)\right]$ and $\Phi$ [a section of $\left.\mathbb{R}^{3} \times \mathfrak{s u}(2)\right]$ are such that (1) $\lim |\Phi|(x) \rightarrow 1$. (2) The value of the Yang-Mills-Higgs functional, $\mathfrak{a}$ of $|x| \rightarrow \infty$ Eq. (5.1), is finite at $(A, \Phi)\}$.

There is a natural topology for $\mathfrak{B}$ with respect to which $\mathfrak{B}=\bigcup_{n} \mathfrak{B}_{n}, n \in \mathbb{Z}[6]$. The critical set of absolute minima of $\mathfrak{a}$ on $\mathfrak{B}_{n}$ are the solutions to the Bogomol'nyi equations [Eq. (5.4)], and every local minimum of $\mathfrak{a}$ on $\mathfrak{B}_{n}$ is an absolute minimum. A non-minimal critical point of $\mathfrak{a}$ in $\mathfrak{B}_{0}$ is known to exist. For this and other possible critical points, the following a priori result applies:

Theorem 1.2. Let $[(A, \Phi)] \in \mathfrak{B}_{n}$ be a solution to the $\mathrm{SU}(2)$ Yang-Mills-Higgs equations on $\mathbb{R}^{3}$ in the Prasad-Sommerfield limit. Then the index, $k$, of the hessian of $\mathfrak{a}$ at $(A, \Phi)$ satiesfies $k \geqq|n|+1$ or else $k=0$ and $(A, \Phi)$ satisfy the Bogomolnyi equations.

The proofs of Theorems 1.1 and 1.2 rely critically on the fact that $\mathbb{R}^{4}$, and $\mathbb{R}^{3} \times S^{1}$ are quaterionic manifolds in the sense that their bundles of anti-self-dual 2 -forms admit three orthonormal, covariantly constant cross-sections. The only compact, oriented 4-manifolds with this property are the 4-torus with its flat metric and a K3 surface with a Yau-metric [7]. On these manifolds, an analog of Theorem 1.1 is true. This is stated as Theorem 3.8.

There are non-compact examples of quaternionic manifolds, for example the self-dual gravitational instanton metrics of Penrose, Gibbons and Hawking, and Hitchin [8]. An analog of Theorem 1.1 should be true for these manifolds also.

It is of course possible that Theorem 1.1 is empty in the sense that there may not be any solutions to the Yang-Mills equations on $S^{4}$ which are not either selfor anti-self-dual. Nonetheless, Theorem 1.1 may still have implications for the topology of the instanton moduli spaces. The theorem suggests the following conjecture:

Conjecture. The inclusion of the moduli space of self-dual connections on a principal $\mathrm{SU}(2)$ bundle $P \rightarrow S^{4}$ of degree $n$ into $\mathfrak{B}_{n}$ induces an isomorphism of the pointed homotopy groups $\pi_{k}(\cdot)$ for $k \leqq 2|n|$.

Indeed, if the Yang-Mills functional satisfied Palais-Smale Condition C [9], then the conjecture would be an immediate corollary to Theorem 1.1. However, the Palais-Smale condition is not satisfied here. A conjecture of this type was made by Atiyah and Jones [4]. 
For the Yang-Mills-Higgs theory, Theorem 1.2 suggests the identical conjecture for the moduli spaces of solutions of the Bogomol'nyi equations, but with the isomorphisms of $\pi_{k}$ true for $k<|n|$. The reader should compare Theorems 1.1 and 1.2 with the results of Eels and Wood on harmonic maps from $C P^{1}$ to $C P^{n}$ (Proposition 9.2 of [10]); the conjecture here has an analogy in the harmonic map problem with the homotopy approximation theorems of Segal in [11].

The first half of this paper, Sects. $2-5$, is an investigation into the formal relationship between the quaternionic structures of $\mathbb{R}^{4}$ (and $\mathbb{R}^{3} \times S^{1}$ ) and the hessian of the Yang-Mills functional. The principal result is Theorem 3.2. The consequences of this theorem for the Yang-Mills equations on $S^{4}$ are discussed in Sect. 4; the consequences for the Yang-Mills-Higgs equations on $\mathbb{R}^{3}$ are discussed in Sect. 5. Sections 6-9 contain the analysis to justify these formal considerations. Of some independent interest is a relative index theorem on $\mathbb{R}^{n}, n \geqq 3$ for Dirac type operators which is stated as Proposition 7.2. The assumptions involved differ considerably from the usual weighted, $L_{2}$ index theorems in the literature; they are tailored for the situations which are typical to gauge theories. The proof occupies Sects. 6 and 7. In Sect. 8, the index theorem is applied to a specific operator arising in Yang-Mills theory. In Sect. 9, the index theorem is applied to the analogous operator in Yang-Mills-Higgs theory on $\mathbb{R}^{3}$. One auxiliary result is a rigorous justification of Weinberg's index calculation in [12].

\section{The Yang-Mills Hessian}

Suppose that $M$ is an oriented Riemannian 4-manifold with no boundary, and that $G$ is a compact, simple Lie group. Let $P \rightarrow M$ be a principal $G$-bundle and denote $\hat{\mathfrak{g}}=P \times{ }_{\text {Ad }} \mathfrak{g}$ and $\mathfrak{g}=\operatorname{Lie} \operatorname{Alg}(G)$. The Yang-Mills action is a functional on the space of smooth connections on $P$ : For a connection $A$ on $P$,

$$
\mathfrak{Y} \mathfrak{M}(A)=\frac{1}{2}\left\|F_{A}\right\|_{2}^{2} \text {. }
$$

Here $F_{A}$ is the curvature of $A$; it is a section of $\hat{\mathrm{g}} \times \hat{2}_{2} T^{*}$, where $T^{*} \rightarrow M$ is the cotangent bundle. The $L_{2}$-norm in Eq. (2.1) is the norm induced by the Riemannian metric on $T^{*}$ and the Killing form on $\mathfrak{g}$.

The curvature of any connection on $P$ satisfies the Bianchi identity,

$$
D_{A} F_{A}=0 \text {. }
$$

A solution to the Yang-Mills equations is a smooth connection, $A$, whose curvature also satisfies

$$
D_{A} * F_{A}=0
$$

Here, $D_{A}: \Gamma\left(\hat{\mathfrak{g}} \otimes \underset{p}{\wedge} T^{*}\right) \rightarrow \Gamma\left(\hat{\mathfrak{g}} \otimes \underset{p+1}{\wedge} T^{*}\right)$ is the covariant exterior derivative that is defined by $A$. The $*$ operator in Eq. $(2.2 \mathrm{~b})$ is the usual Hodge dual.

The hessian at $A$ is the following bilinear form on $\Gamma\left(\hat{\mathrm{g}} \otimes T^{*}\right)$

$$
\mathfrak{H}_{A}(a, b)=\left\langle D_{A} a, D_{A} b\right\rangle_{2}+\left\langle F_{A}, a \wedge b+b \wedge a\right\rangle_{2} .
$$


As $*^{2}=1$ on $\hat{2} T^{*}, *$ induces the decomposition $\hat{2}^{*} T^{*}=P_{+} \hat{\imath}^{*} T^{*}$ $\oplus P_{-} \hat{2}^{*} T^{*}$, where $P_{ \pm}=\frac{1}{2}(1 \pm *)$. Write $F_{ \pm}=P_{ \pm} F_{A}$ and define operators

$$
P_{ \pm} D_{A}: \Gamma\left(\hat{\mathfrak{g}} \otimes T^{*}\right) \rightarrow \Gamma\left(\hat{\mathfrak{g}} \otimes P_{ \pm} \wedge T^{*}\right) .
$$

The hessian as defined above may be written as

$$
\mathfrak{H}_{A}(a, b)=2\left\langle P_{ \pm} D_{A} a, P_{ \pm} D_{A} b\right\rangle_{2}+2\left\langle F_{ \pm}, a \wedge b+b \wedge a\right\rangle_{2} .
$$

Because the Yang-Mills equations are $(\mathfrak{F}=$ Aut $P$ invariant, the hessian has an infinite dimensional null space: If $A$ is a solution to the Yang-Mills equations, then $\mathfrak{H}_{A}(a, \cdot)=0$ for all $a=D_{A} \phi$ with $\phi \in \Gamma(\hat{\mathrm{g}})$.

This pathology can be remedied by considering $\mathfrak{Y M}(\cdot)$ as a functional on the space $\mathfrak{B}$ of isomorphism classes of pairs $(P, A)$ of principal $G$-bundles $P \rightarrow M$ and connections $A$ on $P$. When $M$ is compact, one can do this in a straightforward way by defining $\mathfrak{B}$ as an $L_{k, 2}(k \geqq 2)$ Hilbert manifold as in $[13,14]$. When restricted to the tangent space to an orbit $[A] \in \mathfrak{B}, \mathfrak{H}_{A}(\cdot, \cdot)$ defines a bounded, elliptic bilinear form.

An alternative approach, which is used here, is to consider for a connection $A$ on $P$, the bilinear form

$$
\overline{\mathfrak{H}}_{A}(a, b)=\mathfrak{H}_{A}(a, b)+\left\langle D_{A} * a, D_{A} * b\right\rangle_{2} .
$$

This is an unbounded, symmetric form on $L_{2}\left(\hat{\mathfrak{g}} \otimes T^{*}\right)$; when $M$ is compact, its dense closed domain is $L_{1,2}\left(\hat{\mathrm{g}} \otimes T^{*}\right)$. The associated, formally self-adjoint operator is obtained by integrating by parts in Eq. (2.5): Define $\Delta_{A}$ by

$$
\Delta_{A} a=2 D_{A}^{*} P_{-} D_{A} a+D_{A} D_{A}^{*} a+2 P_{-} F(a) .
$$

Here $D_{A}^{*}: \Gamma\left(\hat{\mathfrak{g}} \otimes \underset{p}{\wedge} T^{*}\right) \rightarrow \Gamma\left(\hat{\mathfrak{g}} \otimes \underset{p-1}{\wedge} T^{*}\right)$ is the formal $L_{2}$-adjoint of $D_{A}$. The zeroth order term in Eq. (2.6) is

$$
P_{-} F(a)=-*\left(F_{-} \wedge a-a \wedge F_{-}\right) .
$$

When $M$ is compact, the index of $\mathfrak{H}_{A}$ is precisely the number of eigenvectors of $\Delta_{A}$ with negative eigenvalue. If $A$ is irreducible, then the nullity of $\Delta_{A}$ and the nullity of $\mathfrak{S}_{A}$ on the tangent space to $[A] \in \mathfrak{B}$ agree.

The index and nullity of $\Delta_{A}$ on $S^{4}$ are restricted by the two theorems below.

Theorem 2.1. Let $G$ be a compact, simple Lie group, and let $P \rightarrow S^{4}$ be a principal $G$-bundle. Let $A$ be a smooth, irreducible solution to the Yang-Mills equations on $P$ which is neither self-dual nor anti-self-dual. Then the number of eigenvectors of $\overline{\mathfrak{H}}_{A}$ with nonpositive eigenvalue is bounded below by $\frac{1}{4}\left|p_{1}(\hat{\mathrm{g}})\right|+2-\operatorname{dim} G$. Here, $p_{1}(\hat{\mathrm{g}})$ is the first Pontrjagin class of $\hat{\mathrm{g}}$.

Theorem 2.2. Let $G=\mathrm{SU}(2)$ or $\mathrm{SU}(3)$ and let $(P, A)$ be as in Theorem 2.1. The number of eigenvectors of $\overline{\mathfrak{H}}_{A}$ with negative eigenvalues is bounded below by $\frac{1}{4}\left|p_{1}(\mathfrak{g})\right|+2$.

Recall that when $G$ is simply connected, the number $p_{1}(\hat{\mathrm{g}})$ classifies, up to isomorphism, the principal $G$-bundles on a compact, oriented 4-manifold without 
boundary. For $G=\mathrm{SU}(2), p_{1}(\hat{\mathrm{g}})=8 k$, where $k$ is the Pontrjagin (instanton) number referred to in Sect. 1. Thus, Theorem 2.2 implies Theorem 1.1. In general, $p_{1}(\hat{\mathrm{g}})$ can be computed using the Chern-Weil formula :

$$
p_{1}(\hat{\mathrm{g}})=\frac{1}{4 \pi^{2}} \int_{M} \operatorname{Tr}_{\mathrm{g}}\left(F_{A} \wedge F_{A}\right),
$$

where $A$ is any connection on $\hat{\mathfrak{g}}$ and $\operatorname{Tr}_{\mathrm{g}}$ is the trace on $\mathfrak{g}$ given by the Killing form.

\section{Formal Aspects}

Crucial to the proofs of the theorems in Sects. 1 and 2 are the two facts: (1) The 4-sphere is conformal to $\mathbb{R}^{4}$ and (2) $\mathbb{R}^{3}$ is $\mathbb{R}^{3} \times S^{1} / S^{1}$. Both $\mathbb{R}^{4}$ and $\mathbb{R}^{3} \times S^{1}$ are "self-dual, gravitational instantons." These are Riemannian 4-manifolds with the property that the bundle $P_{-}{ }_{2} T^{*}$ admits a global, covariantly constant orthonormal frame. On such a 4-manifold, the Riemannian curvature of the metric factors through the traceless endomorphisms of $P_{+} \hat{2}_{2} T^{*}$; i.e., it is Ricci-flat with vanishing anti-self-dual, conformal Weyl tensor. All of the formal manipulations here for $\mathbb{R}^{4}$ and $\mathbb{R}^{3} \times S^{1}$ are possible on such a manifold, cf. Theorem 3.8.

Let $M$ denote $\mathbb{R}^{4}$ or $\mathbb{R}^{3} \times S^{1}$. Let $G$ be a simple, compact Lie group, and let $P \rightarrow M$ be a principal $G$-bundle. Let $\mathfrak{U}=\{$ Smooth, finite Yang-Mills action connections on $P\}$. For notational convenience, denote $\left(P \times_{\text {Ad }} \mathfrak{g}\right) \otimes \wedge_{p} T^{*}$ by $\hat{\mathrm{g}}^{p}$.

For $A \in \mathfrak{A}$, one defines the hessian, $\mathfrak{H}_{A}$, by Eq. (2.4) and one defines via Eq. (2.5) the bilinear form $\overline{\mathfrak{H}}_{A}$. These are both bilinear, symmetric forms which are defined on $\Gamma_{0}\left(\hat{\mathrm{g}}^{1}\right)$ - the set of smooth, compactly supported sections.

Let $\left\{\omega_{i}\right\}_{i=1}^{3}$ be a covariantly constant, orthonormal frame for $P_{-} \wedge T^{*}$. The $\omega^{i}$ define an action of the imaginary quaternions on $T^{*}$ and hence on $\hat{\mathrm{g}}^{1}$. This action is given by

$$
\omega^{i}(a)=\sqrt{2} *\left(\omega^{i} \wedge a\right)
$$

and

$$
\omega^{i}\left(\omega^{j}(a)\right)=-\delta^{i j} a-\varepsilon^{i j k} \omega^{k}(a)
$$

Here, $\varepsilon^{123}=1$ and $\varepsilon^{i j k}$ is completely antisymmetric. Observe that the action is point-wise isometric.

The action preserves the global inner-product $\left\langle\nabla_{A}(\cdot), \nabla_{A}(\cdot)\right\rangle_{2}$ on $\Gamma_{0}\left(\hat{\mathrm{g}}^{1}\right)$. Let $H_{A}$ denote the completion of $\Gamma_{0}\left(\hat{\mathrm{g}}^{1}\right)$ with respect to this innerproduct. The Hilbert space $H_{A}$ is a space of locally $L_{1,2}$ sections of $\hat{\mathrm{g}}^{1}$ (cf. Lemma 6.3). Let $\mathfrak{R}_{A} \subseteq H_{A}$ be a domain for $\overline{\mathfrak{H}}_{A}$ which is invariant with respect to the action in Eq. (3.1).

When exploring the index and nullity of $\overline{\mathfrak{H}}_{A}$, it is useful to consider the restriction of $\overline{\mathfrak{H}}_{A}$ to a particular subspace, $V_{A} \subset \mathfrak{R}_{A}$.

Definition 3.1. Let $A \in \mathfrak{A}$. Define $V_{A}$ by $V_{A}=\left\{a \in \mathfrak{R}_{A}: \delta_{A} a \equiv\left(\sqrt{2} P_{-} D_{A} a, D_{A}^{*} a\right)=0\right\}$.

The relationship between $V_{A}$ and $\overline{\mathfrak{S}}_{A}$ is described in the next theorem, its proof is the subject of the remainder of this section. 
Theorem 3.2. Let $A \in \mathfrak{U}$. If $\operatorname{dim} V_{A}<\infty$, then $\operatorname{dim} V_{A}$ is divisible by 4. Let $E \subset \mathfrak{R}_{A}$ be the maximal subspace on which $\overline{\mathfrak{H}}_{A}$ is negative. If $\operatorname{dim} E \leqq \frac{1}{4} \operatorname{dim} V_{A}$, then there are at least $\frac{1}{4} \operatorname{dim} V_{A}+3-\operatorname{dim} E$ eigenvectors of $\overline{\mathfrak{H}}_{A}$ with zero eigenvalue. Let $G=\mathrm{SU}(2)$ or $\mathrm{SU}(3)$. Suppose that $A$ is a solution to the Yang-Mills equations which is not self-dual. Then $\operatorname{dim} E \geqq \frac{1}{4} \operatorname{dim} V_{A}$.

Proof of Theorem 3.2. As the $\left\{\omega^{i}\right\}$ are covariantly constant, $\omega^{i}$ maps $V_{A}$ into itself. In fact, for $a \in \Gamma\left(\hat{\mathfrak{g}}^{1}\right)$,

$$
\sqrt{2} P_{-} D_{A} \omega^{i}(a)=\omega^{i} D_{A}^{*} a+\varepsilon^{i j k} \omega^{j}\left(\omega^{k}, \sqrt{2} P_{-} D_{A} a\right),
$$

and

$$
D_{A}^{*} \omega^{i} a=-\left(\omega^{i}, \sqrt{2} P_{-} D_{A} a\right) .
$$

Here, an abuse of notation is used to denote by $(\cdot, \cdot)(x)$ the natural pairing $T^{*} \otimes \hat{\mathfrak{g}}^{1} \rightarrow \hat{\mathfrak{g}}^{0}$ which is induced by the metric. Because the $\left\{\omega^{i}\right\}$ acts isometrically, one readily obtains

Lemma 3.3. Let $A \in \mathfrak{U}$. Then $V_{\boldsymbol{A}}$ admits an isometric, left action of the unit quaternions; if $\operatorname{dim} V_{A}<\infty$, then it is a multiple of 4.

Consider the restriction of $\overline{\mathfrak{H}}_{A}$ to the vector space $V_{A}$. This restriction defines a bilinear form $h=\left.\overline{\mathfrak{H}}_{A}\right|_{V_{A}}$ given by

$$
h(a, b)=2\left\langle P_{-} F_{A}, a \wedge b+b \wedge a\right\rangle_{2} \text { for } a, b \in V_{A} .
$$

The trick to proving Theorem 3.2 is to average the value of $h(\cdot, \cdot)$ over the action of the quaternions. (Bourguignon et al. use a similar trick in [3].)

Lemma 3.4. Let $A \in \mathfrak{A}$. Let $h$ be defined as in Eq. (3.5). Then

$$
\sum_{j=1}^{3} h\left(\omega^{j}(a), \omega^{j}(a)\right)=-h(a, a) .
$$

Proof of Lemma 3.4. Observe that for any $a \in \Gamma\left(\hat{\mathfrak{g}}^{1}\right)$, the following equality holds:

$$
\omega^{i} \wedge a \wedge a=2^{-1 / 2} a \wedge *\left(\omega^{i}(a)\right)=-2^{-1 / 2} \omega^{i}(a) \wedge * a .
$$

Now one uses the previous line and Eq. (3.2) to deduce the sequence of equalities below:

$$
\begin{aligned}
\omega^{i} \wedge \omega^{j}(a) \wedge \omega^{j}(a) & =2^{-1 / 2} \omega^{j}(a) \wedge *\left(\omega^{i}\left(\omega^{j}(a)\right)\right), \\
& =-2^{-1 / 2} \omega^{i}(a) \wedge * a-2^{-1 / 2} \varepsilon^{i j k} \omega^{j}(a) \wedge * \omega^{k}(a), \\
& =-2^{-1 / 2} \omega^{i}(a) \wedge * a+2^{-1 / 2} \varepsilon^{i j k} a \wedge *\left(\omega^{j}\left(\omega^{k}(a)\right)\right), \\
& =-2^{-1 / 2} \omega^{i}(a) \wedge * a-2^{-1 / 2} \varepsilon^{i j k} \varepsilon^{j k l} a \wedge * \omega^{l}(a) \\
& =-2^{-1 / 2} a \wedge * \omega^{i}(a)=-\omega^{i} \wedge a \wedge a .
\end{aligned}
$$

As one can expand $P_{-} F_{A}=F_{-}^{i} \omega^{i}$ with $F_{-}^{i} \in \Gamma\left(\hat{\mathfrak{g}}^{0}\right)$, the lemma follows from Eq. (3.7).

Using linear algebra, one can draw from Lemma 3.4 some conclusions about the eigenvalues of $h$ on $V_{A}$. The relevant linear algebra is provided in the next lemma. 
Lemma 3.5. Let $V$ be a real vector space of dimension $4 l$ with metric (, ). Suppose that $V$ admits a left action of the unit quaternions which is isometric. Let $h$ be a symmetric bilinear form which satisfies Eq. (3.6) for all $a \in V$, where $\left\{\omega^{j}\right\}$ is an orthonormal basis for the unit imaginary quaternions. Let $m=$ index of $h$. If $m<l$ then $h$ has at least $4(l-m)$ eigenvectors with eigenvalue zero.

Proof of Lemma 3.5. Let $V_{1}=$ Span \{eigenvectors with negative eigenvalue\}, $V_{2}=\operatorname{Span}\left\{\right.$ orbit of $V_{1}$ under the left action of the unit quaternions and let $V_{3}=V_{2}^{\perp}$. By assumption, $\operatorname{dim} V_{1}=m$, so $\operatorname{dim} V_{2} \leqq 4 m$ and $\operatorname{dim} V_{3} \geqq 4(m-l)$. One must demonstrate that $V_{3} \subset \operatorname{ker} h$. Let $a \in V_{3}$. Then necessarily, $h(a, a)=0$. Indeed if $h(a, a) \neq 0$, then by Eq. (3.6), there exists a unit quaternion $q$ such that $h(q a, q a)<0$. Observe that $a \in V_{3}$ implies $q a \in V_{1}^{\perp}$. However, it is assumed that $h \geqq 0$ on $V_{1}^{\perp}$ so one obtains a contradiction unless $h(a, a)=0$. By polarization, $h(a, b)=0$ for all $b \in V_{3}$. Because $V_{1} \subset V_{3}^{\perp}, h(a, b)=0$ for all $b \in V_{1}$ as well. Finally, consider $h(a, b)$ for $b \in V_{2} \cap V_{1}^{\perp}$. If this were to be nonzero, then by replacing $b$ by $-b$ if necessary, one would have $h(a, b)<0$. Therefore one would have $h(a+\varepsilon b, a+\varepsilon b)<0$ for all $\varepsilon>0$ sufficiently small. However $a+\varepsilon b \in V_{1}^{\perp}$ so it must be true that $h(a+\varepsilon b, a+\varepsilon b) \geqq 0$. The conclusion is that $h(a, b)=0$ for all $b \in V_{2} \cap V_{1}^{\perp}$. Therefore, $V_{3} \subseteq \operatorname{ker} h$ and $\operatorname{dim} \operatorname{ker} h \geqq \operatorname{dim} V_{3} \geqq 4(l-m)$ as claimed.

To summarize, Lemmas 3.3-3.5 imply

Lemma 3.6. Let $A \in \mathfrak{A}$, and let $l=\frac{1}{4} \operatorname{dim} V_{A}$. The quadratic form $\overline{\mathfrak{H}}_{A}$ on $\mathfrak{R}_{A}$ has the following property: There is an $m$ dimensional subspace of $V_{A}$ on which $\overline{\mathfrak{H}}_{A}$ is negative. If $m<l$, there is a subspace $N_{A} \subset V_{A}$ of dimension greater than or equal to $4(l-m)$ such that $\overline{\mathfrak{H}}_{A}(a, b)=0$ for all $b \in V_{A}$ and each $a \in N_{A}$.

In continuing the proof of Theorem 3.2, assume for the sake of argument that the integers, $(m, l)$ of Lemma 3.6 satisfy $m<l$. Consider $\overline{\mathfrak{H}}_{A}$ on any finite dimensional vector space $V_{A} \subset V \subset \mathfrak{R}_{A}$. Let $n_{V}$ be the number of eigenvectors of $\left.\overline{\mathfrak{H}}_{A}\right|_{V}$ with negative eigenvalue. Necessarily, $n_{V} \geqq m$. If $n_{V}-m<4(l-m)$, then $\left.\overline{\mathfrak{H}}_{A}\right|_{V}$ has $4 l-3 m-n_{V}$ eigenvectors in $N_{A}$ with zero eigenvalue. As $V$ can be taken arbitrarily large, one obtains the first statement of the theorem with the observation that when $m<l, 4 l-3 m \geqq l+3$.

To prove the second part of Theorem 3.2, suppose for argument's sake that there is no vector subspace $V \subset \mathfrak{R}_{A}$ of dimension greater than $k$ on which $\overline{\mathfrak{H}}_{A}$ is negative and suppose that $k<l$. Let $\bar{N}_{A}=\left\{a \in V_{A}: \overline{\mathfrak{H}}_{A}(a, \cdot)=0\right\}$. The vector space $\bar{N}_{A}$ is non-empty (see Lemma 3.6). Let $U_{A}=\bar{N}_{A}^{\perp} \cap N_{A}$. The space $U_{A}$ has dimension $\leqq k-m$. Let $\bar{U}_{A}$ denote the span of the orbit of $U_{A}$ under the quaternion action; $\operatorname{dim} \bar{U}_{A} \leqq 4(k-m)$. Note that $\bar{U}_{A} \leqq N_{A}$ and its orthogonal complement $Z_{A} \equiv \bar{U}_{A}^{\perp} \cap N_{A}$ is quaternionic, $\operatorname{dim} Z_{A} \geqq 4(l-k)$ and $Z_{A} \subseteq \bar{N}_{A}$.

When $Z_{A} \neq \emptyset$, one obtains useful information about $P_{-} F_{A}$. In fact, a nonzero element $a \in Z_{A}$ has the property that at each $x \in M$ and for each $i \in(1,2,3)$ and $v=(1, \ldots, 4)$,

$$
\left[F_{-}^{i}(x), a_{v}(x)\right]=0
$$

Here, $F_{-}^{i}=\left(P_{-} F_{A}, \omega^{i}\right) \in \Gamma\left(\hat{\mathrm{g}}^{0}\right)$ and $a_{v}=\frac{\partial}{\partial x^{\nu}}-a \in \Gamma\left(\hat{\mathrm{g}}^{0}\right)$. 
Now restrict to $G=\mathrm{SU}(2)$ or $\mathrm{SU}(3)$. To prove the second part of Theorem 3.2, it is enough to establish that $U_{A}=\emptyset$. Suppose to the contrary that there exists 0 ₹ $a \in Z_{A}$. As neither of the Lie algebras of SU(2) or SU(3) contain the direct sum of two nonabelian subalgebras, one concludes (cf. $[3,15])$ that at each $x \in M$, either

$$
\left[F_{-}^{i}, F_{-}^{j}\right]=0 \text { for all } i, j \in(1,2,3)
$$

or

$$
\left[a_{v}, a_{\mu}\right]=0 \text { for all } \mu, v \in(1,2,3,4) .
$$

If $G=\mathrm{SU}(2)$, both (3.9a, b) must be satisfied everywhere. Since $A$ is a solution to the Yang-Mills equations on $M, A$ is real analytic. As $a \in V_{A}$ is a solution to an elliptic PDE on $M$ with real analytic coefficients, $a$ is also real analytic. The real analytic properties of $A$ and $a$ have as their consequence that if either (3.9a) or (3.9b) is satisfied on an open set $U \subseteq M$, then it is satisfied globally, and so one or the other is globally satisfied. Equation (3.9a) cannot be globally satisfied. Indeed, the YangMills equations imply that $* D_{A} P_{-} F_{A}=0$, and if Eq. (3.9a) were true, the Weitzenböck formula for the operator $P_{-} D_{A} * D_{A}: \Gamma\left(P_{-} \hat{\mathfrak{g}}^{2}\right) \rightarrow \Gamma\left(P_{-} \hat{\mathfrak{g}}^{2}\right)$ would imply that

$$
D_{A} * D_{A} F_{-}^{i}=0, \quad i \in(1,2,3),
$$

where here, $D_{A} * D_{A}: \Gamma\left(\hat{\mathrm{g}}^{0}\right) \rightarrow \Gamma\left(\hat{\mathrm{g}}^{0}\right)$. From Eq. (3.10) and the fact that $P_{-} F_{A} \in L_{2}$, one would conclude that $P_{-} F_{A}$ is covariantly constant - hence zero. This would contradict the assumption that $A$ is not self-dual. Therefore, Eq. (3.9b) must be satisfied everywhere. As $a \in V_{A}$,

$$
\left\|\delta_{A} a\right\|_{2}^{2}=0 .
$$

Due to the imbeddings of $H_{A}$ that are provided by Lemma 6.3, one can integrate by parts in Eq. (3.11) to obtain the identity

$$
0=\left\|\delta_{A} a\right\|_{2}^{2}=\left\|\nabla_{A} a\right\|_{2}^{2} .
$$

There are no curvature terms in the Weitzenböck formula, Eq. (3.12), (cf. $[13,14]$ ) because of Eq. (3.9b). Hence a is covariantly constant and because of Lemma 6.3, $a \equiv 0$. Thus $Z_{A}=\emptyset$ as claimed.

Now consider the case where $M$ is an arbitrary self-dual gravitational instanton. Formally, the preceding arguments are still true. Indeed, $M$ can be covered by a system of coordinates in which the metric is real analytic [16]. The Riemannian curvature terms in the required Weitzenböck formulae conveniently vanish also; so except for the possible existence of covariantly constant sections of $\Gamma\left(\hat{\mathrm{g}}^{0}\right)$, Theorem 3.2 remains true on such $M$. When $M=T^{4}$ with its flat metric, or a K3 surface with a Yau metric, a lower bound for $\operatorname{dim} V_{A}$ can be obtained with the Atiyah-Singer index theorem [17]. In these cases, Theorem 3.2 yields

Theorem 3.8. Let $M$ be smoothly conformal to the flat $T^{4}$ or to a $\mathrm{K} 3$ surface with a Yau metric. Let $G$ be a simple Lie group and let $P \rightarrow M$ be a principal $G$-bundle. Let $A$ be a smooth connection on $P$ and a solution to the Yang-Mills equations. Then the $L_{2}$-index plus nullity of $\overline{\mathfrak{H}}_{A}$ is at least $\frac{1}{4} p_{1}\left(P \times_{\text {Ad }} \mathfrak{g}\right)-\frac{1}{8} \operatorname{dim} G \cdot r(M)+1$, where $r\left(T^{4}\right)=0$ and $r(\mathrm{~K} 3)=8$. 


\section{On $\mathbb{R}^{4}$ and $S^{4}$}

The results of the previous section can be applied to analyze the hessian of Eqs. (2.4) and (2.5) on $\mathbb{R}^{4}$ and also on $S^{4}$. This results in proofs for Theorems 2.1 and 2.2. Before using Theorem 3.2, some technical notions must be introduced to make the theorem less abstract and hence more accessible.

For $A \in \mathfrak{A}$, let $H_{A}$ be the Hilbert space that was introduced in Sect. 3.

The utility of the space $H_{A}$ is in the proposition below, it is the domain where the hessians of Eqs. (2.4) and (2.5) are well defined. This proposition is proved in Sect. 6 .

Proposition 4.1. Let $A \in \mathfrak{A}$. Then

(1) $\overline{\mathfrak{Y}}_{A}$ is a bounded, bilinear form on $H_{A}$.

(2) The number of eigenvectors of $\overline{\mathfrak{H}}_{A}$ with nonpositive eigenvalue is finite.

(3) $\overline{\mathfrak{H}}_{A}$ admits $n$ eigenvectors with negative eigenvalue if and only if there exists a vector subspace $E \subset \mathrm{CH}_{A}$ of dimension $n$ on which $\overline{\mathfrak{G}}_{A}$ is negative.

In order to apply Theorem 3.2 to the form $\overline{\mathfrak{Y}}_{A}$ it is necessary to know the dimension of $V_{A}$ of Definition 3.1.

Proposition 4.2. Let $A \in \mathfrak{A}$. Let $\delta_{A}=\left(\sqrt{2} P_{-} D_{A}, D_{A}^{*}\right): \Gamma\left(\hat{\mathrm{g}}^{1}\right) \rightarrow \Gamma\left(P_{-} \hat{\mathrm{g}}^{2} \oplus \hat{\mathrm{g}}^{0}\right)$, and let $V_{A}=\operatorname{ker} \delta_{A} \cap H_{A}$. Then $\operatorname{dim} V_{A}<\infty$. Define

$$
p_{1}(A)=\frac{1}{4 \pi^{2}} \int_{\mathbb{R}^{4}} \operatorname{Tr}_{\mathfrak{g}}\left(F_{A} \wedge F_{A}\right) .
$$

Then $\operatorname{dim} V_{A} \geqq p_{1}(A)$. If $A$ is a solution to the Yang Mills equations which is not selfdual, then $\operatorname{dim} V_{A} \geqq p_{1}(A)+8$.

The proof of Proposition 4.2 requires an index theorem for the operator $\delta_{A}$. This index theorem is constructed in Sect. 8 where the proof of Proposition 4.3 is given.

The number $p_{1}(A), A \in \mathfrak{A}$ can be computed using a result of Uhlenbeck.

Proposition 4.3 (Uhlenbeck [18]). Let $\mathfrak{s}: S^{4} \backslash\{$ south pole $\} \rightarrow \mathbb{R}^{4}$ be stereographic projection (a conformal equivalence). Let $A$ be a smooth, finite action connection on $\mathbb{R}^{4} \times G$. As bundle with connection, $\left(\mathbb{R}^{4} \times G, A\right)$ is isomorphic to $\left(\mathfrak{s}^{-1}\right)^{*}\left(\tilde{P}, A^{\prime}\right)$, where $\tilde{P} \rightarrow S^{4}$ is a smooth principal G-bundle, and $A^{\prime}$ is an $L_{1,2}$ connection on $\tilde{P}$ which is smooth over $S^{4} \backslash\left\{\right.$ south pole\}. In addition, $p_{1}(A)$ defined by Eq. (4.1) equals $p_{1}\left(\tilde{P} \times_{\mathrm{Ad}} \mathrm{g}\right)$.

The next proposition is now an immediate corollary to Propositions 4.1, 4.2, and Theorem 3.2.

Proposition 4.4. Let $A \in \mathfrak{A}$ on $\mathbb{R}^{4}$. If $\overline{\mathfrak{S}}_{A}$ has $m<\frac{1}{4}\left|p_{1}(A)\right|$ eigenvectors with negative eigenvalues it has at least $\frac{1}{4}\left|p_{1}(A)\right|+3-m$ eigenvectors with zero eigenvalue. Let $G=\mathrm{SU}(2)$ or $\mathrm{SU}(3)$, and suppose that $A$ is a solution to the Yang-Mills equations on $\mathbb{R}^{4}$ which is neither self-dual nor anti-self-dual. Then $\overline{\mathfrak{G}}_{A}$ has at least $\frac{1}{4}\left|p_{1}(A)\right|+2$ eigenvectors with negative eigenvalue.

This last result can be used to analyze the hessians on $S^{4}$ by exploiting the conformal invariance of the Yang-Mills equations. Suppose that $A^{\prime}$ is a smooth 
connection on a bundle $\tilde{P} \rightarrow S^{4}$. It is no loss of generality to assume that $p_{1}(\tilde{\mathfrak{g}}) \geqq 0$, where $\tilde{\mathfrak{g}}=P \times{ }_{\text {Ad }} \mathfrak{g}$. (Here, and in Sect. 8, the symbol “" distinguishes a bundle over $S^{4}$ from a similarly defined bundle on $\mathbb{R}^{4}$, where "^" is used.) Let $\left(\mathbb{R}^{4} \times G, A\right)$ be isomorphic to $\left(\mathfrak{s}^{-1}\right)^{*}\left(\tilde{P}, A^{\prime}\right)$. According to Propositions 4.1 and 4.4 , there exist $m$ eigenvectors $\left\{a_{i}\right\}_{i=1}^{m} \subset H_{A}$ of $\overline{\mathfrak{H}}_{A}$ with negative eigenvalue and if $m<\frac{1}{4} p_{1}(A)$, there exist $n \geqq \frac{1}{4} p_{1}(A)+3-m$ eigenvectors, $\left\{b_{i}\right\}_{i=1}^{n} \subset H_{A} \cap V_{A}$ with zero eigenvalue. The data $\left(\mathbb{R}^{4} \times G, A,\left\{a_{i}\right\},\left\{b_{i}\right\}\right)$ pulls back via $\mathfrak{s}$ to define data $\left(\tilde{P}, A^{\prime},\left\{\tilde{a}_{i}\right\}_{\tilde{z}}\left\{\tilde{b}_{i}\right\}\right)$ on $S^{4}$. Elements in $H_{A}$ pull back via $\mathfrak{s}$ to elements in $L_{1,2}\left(\tilde{\mathfrak{g}}^{1}\right)$. Therefore $\left\{\tilde{a}_{i}, \tilde{b}_{j}\right\} \subset L_{1,2}\left(\tilde{\mathfrak{g}}^{1}\right)$. This space is a maximal domain for the Hessian, $\mathfrak{H}_{A^{\prime}}$ and for $\overline{\mathfrak{H}}_{A^{\prime}}$. The Hessian is conformally invariant in the sense that

$$
\mathfrak{S}_{A^{\prime}}(\tilde{a}, \tilde{b})=\mathfrak{H}_{A}(a, b)
$$

for all $a, b \in H_{A}$. However, $\overline{\mathfrak{S}}_{A}$ is not conformally invariant.

Suppose that $A^{\prime}$ is also a solution of the Yang-Mills equations on $S^{4}$. In this case, $\mathfrak{H}_{A^{\prime}}$ has the property that for any $a, b \in L_{1,2}\left(\tilde{\mathfrak{g}}^{1}\right)$ and $\phi \in L_{2,2}(\tilde{\mathfrak{g}})$,

$$
\mathfrak{H}_{A^{\prime}}\left(a+D_{A} \phi, b\right)=\mathfrak{H}_{A^{\prime}}(a, b) \text {. }
$$

In order to exploit the above invariances, one uses the fact that for each $a \in L_{1,2}\left(\tilde{\mathfrak{g}}^{1}\right)$, there exists a unique $\phi \in L_{2,2}(\tilde{\mathfrak{g}}) \cap\left(\operatorname{ker} D_{A^{\prime}}\right)^{\perp}$ which satisfies $(\mathrm{cf} .[13,14])$

$$
D_{A^{\prime}}^{*} a+D_{A^{\prime}}^{*} D_{A^{\prime}} \phi=0 .
$$

This fact allows one to conclude that for each $i \in(1, \ldots, m)$, and $j \in(1, \ldots, n)$ there exists a unique $\phi_{i}\left(\right.$ and $\left.\phi_{j}\right) \in L_{2,2}(\tilde{\mathfrak{g}}) \cap\left(\operatorname{ker} D_{A^{\prime}}\right)^{\perp}$ such that $\bar{a}_{i}=\tilde{a}_{i}+D_{A^{\prime}} \phi_{i}$ (and $\bar{b}_{j}=\tilde{b}_{j}$ $\left.+D_{A^{\prime}} \phi_{j}\right)$ satisfies $D_{A^{\prime}}^{*} \bar{a}_{i}=0\left(D_{A^{\prime}}^{*}, \bar{b}_{j}=0\right)$.

Equations (4.2) and (4.3) imply that the set $\left\{\bar{a}_{i}\right\}_{i=1}^{m}$ span a vector space in $L_{1,2}\left(\tilde{\mathfrak{g}}^{1}\right)$ on which $\overline{\mathfrak{H}}_{A^{\prime}}$ is negative. Standard arguments imply at least $m$ eigenvectors of $\overline{\mathfrak{H}}_{A^{\prime}}$ exist with negative eigenvalue. With Proposition 4.4 , one obtains Theorem 2.2.

In order to obtain Theorem 2.1, one must investigate the set $\left\{\bar{b}_{j}\right\}_{j=1}^{n}$. Each $\bar{b}_{j}$ is a eigenvector of $\overline{\mathfrak{H}}_{A^{\prime}}$ on $S^{4}$ with zero eigenvalue. However, the set $\left\{\bar{b}_{j}\right\}_{j=1}^{n}$ is not necessarily linearly independent. This can happen if and only if there is a linear combination from $\left\{\tilde{b}_{j}\right\}_{\tilde{c}}$ which is equal to $D_{A^{\prime}} \tilde{\psi}$ for some $\tilde{\psi} \in L_{2,2}(\tilde{\mathrm{g}})$. If $\tilde{b} \in \operatorname{Span}\left\{\tilde{b}_{j}\right\}$ is such a vector, then $\tilde{b}$ pulls back to $\mathbb{R}^{4}$ via inverse stereo-graphic projection to $b=D_{A} \psi \in V_{A}$ where $\psi \in \Gamma\left(\mathbb{R}^{4} \times \mathfrak{g}\right)$. Using the fact that $\delta_{A}\left(D_{A} \psi\right)=0$ one obtains the following lower bound for $\operatorname{dim} \operatorname{Span}\left\{\bar{b}_{j}\right\}$.

Lemma 4.5. Let $\left(\tilde{P}, A^{\prime}\right)$ be a smooth pair consisting of a principal $G$-bundle $\tilde{P} \rightarrow S^{4}$ and a smooth connection, $A^{\prime}$ on $\tilde{P}$. Let $\left(\mathbb{R}^{4} \times G, A\right)$ be isomorphic to $\left(\mathfrak{s}^{-1}\right)^{*}\left(\tilde{P}, A^{\prime}\right)$. Let $K=\left\{\psi \in \Gamma\left(\mathbb{R}^{4} \times \mathfrak{g}\right): D_{A} \psi \in V_{A}\right\}$. Then $\operatorname{dim} K \leqq \operatorname{dim} G$.

The lemma is proved in Sect. 8. Observe that Proposition 4.4 and Lemma 4.5 imply Theorem 2.1 .

\section{The Yang-Mills-Higgs Equations}

The Yang-Mills-Higgs equations on $\mathbb{R}^{3}$ (with no Higgs potential) are identical to the static Yang-Mills equations on $\mathbb{R}^{3} \times S^{1}$. (See, e.g., [5], and [6] for a lengthy 
discussion of this system.) The circle acts on $\mathbb{R}^{3} \times S^{1}$ in the obvious way. Let $P^{\prime} \rightarrow \mathbb{R}^{3} \times S^{1}$ be a principal $G$-bundle; one can consider connections on $P^{\prime}$ which are finite action solutions to the Yang-Mills equations on $\mathbb{R}^{3} \times S^{1}$ and which are invariant with respect to the aforementioned circle action (cf. [19]). Such an invariant connection is uniquely defined by a pair $c=(A, \Phi)$, where $A$ is a connection on $P=\mathbb{R}^{3} \times G$ and $\Phi$ is a section of $\hat{\mathfrak{g}}=\mathbb{R}^{3} \times \mathfrak{g}$. The action of an invariant connection $c=(A, \Phi)$ reduces to the following integral over $\mathbb{R}^{3}$ :

$$
\mathfrak{a}(c)=\frac{1}{2} \int_{\mathbb{R}^{3}}\left\{\left|F_{A}\right|^{2}+\left|D_{A} \Phi\right|^{2}\right\} d^{3} x .
$$

This functional is considered as a functional on

$$
\mathfrak{C}=\left\{\text { Smooth } c=(A, \Phi): \mathfrak{a}(c)<\infty \text { and } \lim _{|x| \rightarrow \infty}|\Phi|(x) \rightarrow 1\right\}
$$

A critical point of $\mathfrak{a}, c \in \mathbb{C}$, satisfies the Yang-Mills equations on $\mathbb{R}^{3} \times S^{1}$; these are equivalent to the Yang-Mills-Higgs equations on $\mathbb{R}^{3}$ :

$$
\begin{gathered}
D_{A}^{*} D_{A} \Phi=0, \\
D_{A}^{*} F_{A}+\left[\Phi, D_{A} \Phi\right]=0 .
\end{gathered}
$$

The formal adjoints $D_{A}^{*}$ are defined with respect to the $L_{2}$ inner-products on $\Gamma\left(\hat{\mathfrak{g}} \otimes \hat{p}_{p} T^{*}\left(\mathbb{R}^{3}\right)\right)$. The self-duality equations on $\mathbb{R}^{3} \times S^{1}$ for an $S^{1}$-invariant connection given by $c=(A, \Phi)$ are called the Bogomol'nyi equations :

$$
F_{A}= \pm * D_{A} \Phi \text {. }
$$

Here, $*: \hat{p}_{p} T^{*}\left(\mathbb{R}^{3}\right) \rightarrow \underset{3-p}{\wedge} T^{*}\left(\mathbb{R}^{3}\right)$ is the Hodge dual on $\mathbb{R}^{3}$.

The motivation for introducing $\mathfrak{a}$ and Eqs. (5.3) and (5.4) as the $S^{1}$ invariant Yang-Mills equations on $\mathbb{R}^{3} \times S^{1}$ is that $\mathbb{R}^{3} \times S^{1}$ is a self-dual gravitational instanton. The action of $\Gamma\left(P_{-} \hat{2}_{2} T^{*}\left(\mathbb{R}^{3} \times S^{1}\right)\right)$ on $\Gamma\left(T^{*}\left(\mathbb{R}^{3} \times S^{1}\right)\right)$ respects the $S^{1}$ invariant sections. Therefore, the formal results in Sect. 3 can be used to analyze the hessian of $a$ at a solution $c=(A, \Phi)$ to Eq. (5.3).

In the interest of brevity, only the case $G=\mathrm{SU}(2)$ will be discussed from this point on.

Prior to presenting the results of the analysis of the hessian, certain auxiliary facts and definitions must be introduced. The definitions will be given in terms that are intrinsic to $\mathbb{R}^{3}$; it should be apparent what the corresponding $\mathbb{R}^{3} \times S^{1}$ definitions are, as given in Sect. 3.

To begin, it should be pointed out that the space $\mathbb{C}$ is naturally topologized in such a way that it is the disjoint union of path components, $\mathfrak{C}_{n}, n \in \mathbb{Z}[6]$. The integer $n$ is the degree of the map $\Phi /|\Phi|$ as a map from a 2-sphere of large radius in $\mathbb{R}^{3}$ to the unit 2-sphere in $\mathfrak{s} \mathfrak{l}(2)$. A lower bound for the index of the hessian of $\mathfrak{a}$ at $c$ will be given in terms of this integer $n$.

It is convenient to explicitly introduce the quaternionic structure of Sect. 3. Let $\left\{\tau^{j}\right\}_{j=1}^{3}$ be an orthonormal basis for the imaginary quaternions with $\tau^{j *}=-\tau^{j}$ and $\tau^{i} \tau^{j}=-\delta^{i j}+\varepsilon^{i j k} \tau^{k}$. The vector bundle $\hat{\mathfrak{g}} \otimes\left(T^{*}\left(\mathbb{R}^{3}\right) \oplus \mathbb{R}\right)$ is isomorphic to $\hat{\mathfrak{g}}^{\prime} \equiv \hat{\mathfrak{g}} \times \mathbb{H}$, 
where $\mathbb{H}$ is the quaternions. This isomorphism sends $\psi=(a, \phi)$ to $\psi=a_{k} \tau^{k}+\phi$, where $a_{k}=\frac{\partial}{\partial x^{k}}-a \in \Gamma(\hat{\mathfrak{g}})$.

Corresponding to the space $H_{A}$ of Sects. 3 and 4, one has

Definition 5.1. Let $c=(A, \Phi) \in \mathfrak{C}$. Define $H_{c}$ to be the completion of $\Gamma_{0}\left(\hat{\mathrm{g}}^{\prime}\right)$ in the norm defined by the metric

$$
\langle\psi, \chi\rangle_{c}=\left\langle\nabla_{A} \psi, \nabla_{A} \chi\right\rangle_{2}+\langle[\Phi, \psi],[\Phi, \chi]\rangle_{2} .
$$

The bilinear form $\overline{\mathfrak{H}}_{c}$ at $c=(A, \Phi) \in \mathbb{C}$ is defined initially on $\Gamma_{0}\left(\hat{\mathfrak{g}}^{\prime}\right)$ to be

$$
\overline{\mathfrak{S}}_{c}\left(\psi_{1}, \psi_{2}\right)=\left\langle\psi_{1}, \psi_{2}\right\rangle_{c}+2\left\langle F_{A}, a_{1} \wedge a_{2}+a_{2} \wedge a_{1}\right\rangle_{2}+2\left\langle D_{A} \Phi,\left[a_{1}, \phi_{2}\right]+\left[a_{2}, \phi_{1}\right]\right\rangle
$$

where $\psi_{i}=\left(a_{i}, \phi_{i}\right) \in \Gamma_{0}\left(\hat{\mathrm{g}}^{\prime}\right)$ for $i=1,2$.

The analog of Proposition 4.1 follows; it is proved in Sect. 6.

Proposition 5.2. Let $c \in \mathfrak{C}$. Then

(1) $\overline{\mathfrak{H}}_{c}$ is a bounded, bilinear form on $H_{c}$.

(2) The number of eigenvectors of $\overline{\mathfrak{H}}_{c}$ with nonpositive eigenvalue is finite.

(3) $\overline{\mathfrak{H}}_{c}$ admits $n$ eigenvectors with negative eigenvalue if and only if there exists a vector subspace $E \subset H_{c}$ of dimension $n$ on which $\overline{\mathfrak{H}}_{c}$ is negative.

The number of negative eigenvalues of $\overline{\mathfrak{H}}_{c}$ for $c \in \mathfrak{C}_{n}$ a solution to Eq. (5.3) is stated in Theorem 1.2. If $c \in \mathfrak{C}_{n}$ is not a solution to Eq. (5.3), one has

Theorem 5.3. Let $c \in \mathfrak{C}_{n}$. If $\overline{\mathfrak{H}}_{c}$ has $m<|n|$ eigenvectors with negative eigenvalue, it has at least $|n|-m+3$ eigenvectors with zero eigenvalue.

Proof of Theorems 1.2 and 5.3. The formal aspects of the proof are provided in Sect. 3 ; it is simply a matter to check that the results therein have the proper $S^{1}$-equivariance. In proving the theorems, one can restrict to the case $n \geqq 0$ with no loss of generality.

In the $n \geqq 0$ case, the $S^{1}$-equivalent version of Definition 3.1 is

Definition 5.4. Let $c \in \mathfrak{C}$. Define the first order operator $\mathfrak{D}_{c}: \Gamma\left(\hat{\mathfrak{g}}^{\prime}\right) \rightarrow \Gamma\left(\hat{\mathfrak{g}}^{\prime}\right)$ by

$$
\mathfrak{D}_{c}=-\tau^{j}\left(\nabla_{A}\right)_{j}+[\Phi, \cdot] \text {. }
$$

Define $V_{c}=\operatorname{ker} \mathfrak{D}_{c} \cap H_{c}$.

Observe that the unit quaternions act on $V_{c}$ isometrically by multiplication from the right, $\psi \rightarrow \psi h$ for $\psi \in V_{c}$ and $h \in \mathbb{H}$. This is the action described by Lemma 3.3.

The dimension of $V_{c}$ is bounded from below using an index theorem for operator $\mathfrak{D}_{c}$.

Proposition 5.5. Let $n \geqq 0$ and let $c \in \mathfrak{C}_{n}$. Then $4 n \leqq \operatorname{dim} V_{c}<\infty$. If $c$ is also a solution to Eq. (5.3) but if $c$ is not a solution to Eq. (5.4) then $4(n+1) \leqq \operatorname{dim} V_{c}$.

The proof of this last proposition is technical, and it is provided in Sect. 9.

One must now check that the proof of Theorem 3.2 can be done in the $S^{1}$-equivariant context; this is straightforward and omitted here. Observe that Theorems 1.2 and 5.3 are direct consequences of Proposition 5.5 and Theorem 3.2. 


\section{Quadratic Forms}

In order to prove Propositions 4.1 and 5.2 it is first necessary to investigate the properties of certain quadratic forms on the space of sections of a vector bundle over $\mathbb{R}^{n}, n=3,4$. It is not a lot of extra work to consider the more general situation below.

Consider a quadratic form, $Q$, of the following type:

$$
Q(a, b)=\langle a, b\rangle_{Q}+\langle a, \mathfrak{R}(b)\rangle_{2} \text {. }
$$

Here, $a, b$ are compactly supported sections of a Riemannian vector bundle $\zeta \rightarrow \mathbb{R}^{n}$, $n>2$ with finite dimensional fiber, $V ; \mathfrak{R} \in \Gamma(\operatorname{End} \zeta) ;\langle\cdot, \cdot\rangle_{2}$ denotes the $L_{2}$-inner product on $\Gamma(\zeta)$; and $\langle\cdot, \cdot\rangle_{Q}$ is a metric on $\Gamma(\zeta)$. The metric $\langle\cdot, \cdot\rangle_{Q}$ is assumed of the form

$$
\langle a, b\rangle_{Q}=\left\langle\nabla_{A} a, \nabla_{A} b\right\rangle_{2}+\langle q(a), q(b)\rangle_{2}
$$

with $q \in \Gamma($ End $\zeta)$ and $A$ is a smooth, metric compatible connection on $\zeta$. The following restrictions on $q$ and $\mathfrak{R}$ are required:

Definition 6.1. A section $\mathfrak{R} \subset \Gamma($ End $\zeta)$ satisfies Property ${ }^{*}$ with respect to a metric $\langle\cdot, \cdot\rangle_{Q}$ on $\Gamma(\zeta)$ if the following is true:

Let $\chi_{r}$ denote the characteristic function of the set $B_{r}=\left\{x \in \mathbb{R}^{n}:|x|<r\right\}$. Property $*$ is satisfied if, given $\varepsilon>0$, there exists $r=r(\varepsilon)<\infty$ such that for all compactly supported $a, b \in \Gamma(\zeta)$,

$$
\left|\left\langle\left(1-\chi_{r}\right) a, \mathfrak{R}(b)\right\rangle_{2}\right| \leqq \varepsilon\langle a, a\rangle_{Q}^{1 / 2}\langle b, b\rangle_{Q}^{1 / 2} .
$$

A quadratic form $Q$ is said to be admissible if it can be written as in Eqs. (6.1) and (6.2) such that $\mathfrak{R}$ satisfies Property $*$ with respect to $\langle\cdot, \cdot\rangle_{Q}$.

After investigating the properties of quadratic forms which are admissible, the Yang-Mills and Yang-Mills-Higgs theories are used to provide some natural examples of admissible forms. The section ends with the proofs of Propositions 4.1 and 5.2.

Henceforth, $Q$ will denote an admissible quadratic form. For discussing the properties of $Q$, some convenient function spaces are provided in the next definition.

Definition 6.2. Let $\Gamma_{0}(\zeta)$ denote the space of compactly supported sections of $\zeta$. Define $H_{Q}$ as the Hilbert space completion of $\Gamma_{0}(\zeta)$ in the inner product $\langle a, b\rangle_{Q}$.

The lemma below states that the elements in $H_{Q}$ are sedtions of $\zeta$.

Lemma 6.3. Let $\psi \in H_{Q}$. Then

$$
\left\|\nabla_{A} \psi\right\|_{2} \geqq \frac{n-2}{n-1}\|\psi\|_{\frac{2 n}{n-2}} .
$$

If $\alpha<n-2$, then

$$
\left\|\left(1+|x|^{2}\right)^{-\alpha / 2} \nabla_{A} \psi\right\|_{2} \geqq \frac{(n-\alpha-2)}{2}\left\|\left(1+|x|^{2}\right)^{-\frac{(1+\alpha)}{2}} \psi\right\|_{2} .
$$

Proof of Lemma 6.3. To establish Eq. (6.4), observe that because the connection, $A$, is metric compatible, one has for smooth $a$ the pointwise inequality 


$$
\left|\nabla_{A} a\right|(x) \geqq|d| a||(x) \text { a.e. }
$$

The first assertion of the lemma follows from Eq. (6.5), cf. Chap. 2 of [20]. The second assertion also follows from Eq. (6.5); but for numerical constants, the proof is identical to the proof of Lemma 5.4 in [21].

Lemma 6.4. The form $Q$ extends to a smooth, bounded, symmetric form on $H_{Q}$.

Proof of Lemma 6.4. The extension of $Q$ to $H_{Q}$ is an immediate consequence of Eq. (6.3) and Lemma 6.3.

It is worth remarking here that $Q$ defines an unbounded, symmetric form on $L_{2}\left(\zeta ; \mu d^{n} x\right)$, where $\mu \in C^{\infty}\left(\mathbb{R}^{n}\right)$ satisfies the growth condition $\mu(x) \geqq$ const $|x|^{-2}$. A fact which is not proved here is that $H_{Q} \cap L_{2}\left(\zeta ; \mu d^{n} x\right)$ is the maximal closed domain for $Q$ on $L_{2}\left(\zeta ; \mu d^{n} x\right)$.

Equation (6.3) implies the following useful inequality: Given $\varepsilon>0$, there exists $c(\varepsilon)<\infty$ such that for all $a \in H_{Q}$,

$$
(1-\varepsilon)\langle a, a\rangle_{Q} \leqq Q(a, a)+c(\varepsilon)\left(\sup _{|x|<r(\varepsilon)}|\mathfrak{R}(x)|\right)\left\langle\chi_{r(\varepsilon)} a, a\right\rangle_{2} .
$$

Consider the eigenvalues of $Q$.

Definition 6.5. An element $a \in H_{Q}$ has eigenvalue $E$ if for all $b \in H_{Q}$,

$$
Q(a, b)=E\langle a, b\rangle_{Q} .
$$

Concerning nonpositive eigenvalues, one has

Lemma 6.6. Let $Q$ be an admissible form. Then (1) the number of eigenvectors of $Q$ with nonpositive eigenvalue is finite; and (2) there exists a vector space $E \subset H_{Q}$ on which $Q$ is negative if and only if the number of eigenvectors of $Q$ with negative eigenvalue is greater than $\operatorname{dim} E$.

Proof of Lemma 6.6. The proof of (1) is strategically like the proof of Theorem 3.2 in [22]. Let $a \in H_{Q}$ be an eigenvector with nonpositive eigenvalue. Observe that due to Eqs. (6.6) and (6.7), for any $R>0$

$$
1 \leqq\|a\|_{Q}^{2} \leqq z\left\langle\chi_{R} a, a\right\rangle_{2},
$$

where $z<\infty$ is independent of $a$. Generality has not been lost by setting $\|a\|_{Q}^{2}=1$. Observe that if $a$ is a convex linear combination of $H_{Q}$-eigenvectors, $\left\{a_{i}\right\}$ with nonpositive eigenvalues, then $a$ also satisfies Eq. (6.8). Each eigenvector $a_{i}$ is a solution to an elliptic, 2nd order PDE on $B_{R+1}$. Thus, one obtains with the Friedrichs Lemma (cf. Theorem 3.5 of [22], also [23]) and Lemma 6.3 the estimate

$$
\left\|a_{i}\right\|_{C^{2} ; B_{R}} \leqq z_{1}\left\|a_{i}\right\|_{2 ; B_{R+1}} \leqq z_{2}\|a\|_{Q}=z_{2} .
$$

Here, $z_{1}$ and $z_{2}$ are constants which depend on the eigenvalue of $a_{i}$, and on $R$. As $Q(\cdot, \cdot)$ is bounded from below, and since the eigenvalue of $a_{i}$ is assumed to be nonpositive, the constant $z_{2}$ is independent of $a_{i}$. Equation (6.9) is also valid when $a$ is a convex linear combination of eigenvectors $a_{i}$ with nonpositive eigenvalue. At this junction, the Gromov and Lawson argument is valid word for word: Choose an $\varepsilon$-dense set $\left\{x_{m}\right\}_{m=1}^{N}$ for $B_{R}$ (each $x \in B_{R}$ is within $\varepsilon$ of some $x_{m}$ ). If the number of 
eigenvectors with nonpositive eigenvalue is greater than $N \operatorname{dim} V$, there exists a convex linear combination $a=\sum_{i} \alpha_{i} a_{i}$ of $\hat{L}$-eigenvectors, $a_{i}$, with nonpositive eigenvalue which vanishes on each $x_{m}$. Thus, for all $x \in B_{R},|a|(x) \leqq z_{2} \varepsilon$ and so $\|a\|_{2 ; B_{R}} \leqq\left|B_{R}\right| z_{1} \varepsilon$. For $\varepsilon$ sufficiently small, this contradicts Eq. (6.9). This establishes an upper bound which proves Statement (1) of Lemma 6.6.

Proof of Statement (2) of Lemma 6.6. The if part is obvious. The only if part is a standard proof by induction: Suppose there exists $l(\geqq 0)$ eigenfunctions $\left\{a_{i}\right\}_{i=1}^{l}$ of $Q$ with negative eigenvalue and suppose that there exists $a \in H_{Q}$ which is orthogonal to $\left\{a_{i}\right\}_{i=1}^{l}$ and $Q(a, a)<0$. Statement (2) follows when it is established that an eigenvector $a_{l+1}$ exists with negative eigenvalue which is orthogonal to $\left\{a_{i}\right\}_{i=1}^{l}$. The existence of $a_{l+1}$ is proved by minimizing $Q$ over $H^{l}=\left\{b \in H_{Q}:\|b\|_{H_{Q}}=1\right.$ and $\left\langle b, a_{i}\right\rangle_{Q}=0$ for $\left.i \in(1, \ldots, l)\right\}$. The weak convergence in $H_{Q}$ of a minimizing sequence, $\left\{b_{\alpha}\right\} \subset H^{l}$ for $Q$ is guaranteed by Eq. (6.6), and Lemma 6.3. Let $b$ denote a weak limit. The weak convergence implies that

$$
\left\langle b, a_{i}\right\rangle_{Q}=0 \text { for } i \in(1, \ldots, l),
$$

and

$$
\langle b, b\rangle_{Q} \leqq \lim _{\alpha \rightarrow \infty}\left\langle b_{\alpha}, b_{\alpha}\right\rangle_{Q} .
$$

The fact that $\Re$ satisfies Property * [see Eq. (6.3)] implies that the function $a \mapsto\langle a, \mathfrak{R}(a)\rangle_{2}$ from $H_{Q}$ to $\mathbb{R}$ is weakly continuous. Thus

$$
Q(b, b) \leqq \lim _{\alpha \rightarrow \infty} Q\left(b_{\alpha}, b_{\alpha}\right)<0 .
$$

From Eq. (6.11), one concludes that $b \neq 0$. The vector $\|b\|_{Q}^{-1} b \in H^{l}$; therefore Eq. (6.11) implies a priori that $Q(b, b)=\inf _{H^{l}} Q(\cdot, \cdot)$ and that $\|b\|_{Q}=1$. The conclusion is that $Q$ takes on its minimum on $H^{l}$ at $b$. The differentiability of $Q$ on $H_{Q}$ implies that $a_{l+1}=b$ is the required eigenvector.

As a parenthetical remark, it is worth noting that the number of eigenvectors of $Q$ with negative eigenvalue is the same as the number of $L_{2}\left(\zeta ; \mu d^{n} x\right)$ eigenvectors of $Q$ with negative eigenvalue when $Q$ is considered as an unbounded form on $L_{2}\left(\zeta ; \mu d^{n} x\right)$. Here as before, $\mu(x) \geqq$ const $|x|^{-2}$ is a smooth function.

Examples of admissible forms are given by the following lemmas.

Lemma 6.7. Let $A \in \mathfrak{U}$ on $\mathbb{R}^{4}$. Let $\delta_{A}$ be as defined in Proposition 4.2, and let $\delta_{A}^{*}$ denote its formal $L_{2}$-adjoint. The quadratic forms $\left\langle\delta_{A}^{*}(\cdot), \delta_{A}^{*}(\cdot)\right\rangle_{2}$ on $\Gamma_{0}\left(P_{-} \hat{\mathfrak{g}}^{2} \oplus \hat{\mathfrak{g}}^{0}\right)$ and $\left\langle\delta_{A}(\cdot), \delta_{A}(\cdot)\right\rangle_{2}$ and $\overline{\mathfrak{H}}_{A}$ on $\Gamma_{0}\left(\hat{\mathfrak{g}}^{1}\right)$ are admissible.

Lemma 6.8. Let $c=(A, \Phi) \in \mathfrak{C}$ be as defined in Sect. 5. Let $\mathfrak{D}_{c}$ be as in Definition 5.4, and let $\mathfrak{D}_{c}^{*}$ be its formal $L_{2}$ adjoint. The quadratic forms $\left\langle\mathfrak{D}_{c}(\cdot), \mathfrak{D}_{c}(\cdot)\right\rangle_{2}$, $\left\langle\mathfrak{D}_{c}^{*}(\cdot), \mathfrak{D}_{c}^{*}(\cdot)\right\rangle_{2}$ and $\overline{\mathfrak{G}}_{\mathrm{c}}$ on $\Gamma_{0}\left(\hat{\mathfrak{g}}^{\prime}\right)$ are all admissible.

Proof of Lemma 6.7. One can write each of the three quadratic forms of the lemma as follows: Denote the form in question by $Q$. Then for compactly supported $\psi_{1}, \psi_{2}$,

$$
Q\left(\psi_{1}, \psi_{2}\right)=\left\langle\nabla_{A} \psi_{1}, \nabla_{A} \psi_{2}\right\rangle_{2}+\left\langle\psi_{1}, F_{Q}\left(\psi_{2}\right)\right\rangle_{2},
$$

where $F_{Q}(\cdot)$ in each case is a matrix with coefficients which are linear combinations of the components of the curvature of $F_{A}$ for $A \in \mathfrak{U}$. Equation (6.12) is 
derived from the Weitzenböck formulas for $\delta_{A}$ and $\delta_{A}^{*}$ in $[13,14]$. Hence, each $Q$ in question is of the kind described in Eq. (6.1). To establish that $F_{Q}$ satisfies Property * with respect to the norm $\left\|\nabla_{A}(\cdot)\right\|_{2}$, one uses the fact that $F_{A} \in L_{2}$. Indeed, given $\varepsilon>0$, there exists $r(\varepsilon)<\infty$ such that $\left\|\left(1-\chi_{r}\right) F_{A}\right\|_{2}<\varepsilon$. Thus, for compactly supported $\psi_{1}, \psi_{2}$

$$
\begin{aligned}
\left\langle\left(1-\chi_{r}\right) \psi_{1}, F_{Q}\left(\psi_{2}\right)\right\rangle_{2} \mid & \leqq \text { const }\left\|\left(1-\chi_{r}\right) F_{A}\right\|_{2}\left\|\psi_{1}\right\|_{4}\left\|\psi_{2}\right\|_{4}, \\
& \leqq \varepsilon \text { const }\left\|\nabla_{A} \psi_{1}\right\|_{2}\left\|\nabla_{A} \psi_{2}\right\|_{2} .
\end{aligned}
$$

The first line above uses Hölder's inequality, and the second line uses Lemma 6.3. Hence Property * is satisfied by $F_{Q}(\cdot)$ as required.

Proof of Lemma 6.8. The form $\overline{\mathfrak{H}}_{c}$ is given in Eq. (5.6), and the other forms in question can be re-expressed as follows:

$$
\begin{aligned}
\left\langle\mathfrak{D}_{c} \psi_{1}, \mathfrak{D}_{c} \psi_{2}\right\rangle_{2} & =\left\langle\psi_{1}, \psi_{2}\right\rangle_{c}+\left\langle\psi_{1}, G_{-}\left(\psi_{2}\right)\right\rangle_{2}, \\
\left\langle\mathfrak{D}_{c}^{*} \psi_{1}, \mathfrak{D}_{c}^{*} \psi_{2}\right\rangle_{2} & =\left\langle\psi_{1}, \psi_{2}\right\rangle_{c}-\left\langle\psi_{1}, G_{+}\left(\psi_{2}\right)\right\rangle_{2},
\end{aligned}
$$

where $\psi_{i}=\left(a_{i}, \phi_{i}\right) \in \Gamma_{0}\left(\hat{\mathrm{g}}^{\prime}\right)$ for $i=1,2$ and

$$
G_{ \pm}(\psi) \equiv \tau_{k}\left(\frac{1}{2} \varepsilon^{i j k}\left[F_{i j}, \psi\right] \mp\left[\nabla_{A} \Phi_{k}, \psi\right]\right) .
$$

Hence each form is of the kind given in Eq. (6.1). The proof of admissibility requires additionally, Lemma 6.6 of [6]. Using this result, the proof of Lemma 6.8 is completely analogous to the proof of Lemma 6.7 and it is left to the reader.

Proof of Proposition 4.1 and 5.2. The propositions follow immediately from Lemmas 6.4, and 6.6 given a priori Lemmas 6.7 and 6.8, respectively.

\section{Index Theorems}

In both Proposition 4.2 and Proposition 5.5, one is required to compute the index of a first order elliptic operator $\mathfrak{D}: \Gamma(\zeta) \rightarrow \Gamma\left(\zeta^{\prime}\right)$, where $\zeta, \zeta^{\prime} \rightarrow \mathbb{R}^{n}$ are vector bundles with finite dimensional fibers $V, V^{\prime}$, respectively. In both cases, the operator $\mathfrak{D}$ has the property that the quadratic form

$$
Q_{\mathfrak{D}}(\cdot, \cdot)=\langle\mathfrak{D}(\cdot), \mathfrak{D}(\cdot)\rangle_{2}
$$

is of the type discussed in Sect. 6, Eq. (6.1); and in both cases, Property $*$ of Definition 6.1 is satisfied (see Lemmas 7.3 and 7.4).

Definition 7.1. Let $\mathfrak{D}: \Gamma(\zeta) \rightarrow \Gamma\left(\zeta^{\prime}\right)$ be a first order, elliptic operator with smooth coefficients. Let $\mathfrak{D}^{*}: \Gamma\left(\zeta^{\prime}\right) \rightarrow \Gamma(\zeta)$ denote the formal $L_{2}$-adjoint of $\mathfrak{D}$. The operator $\mathfrak{D}$ is admissible provided that both $Q_{\mathfrak{D}}$ and $Q_{\mathfrak{D} *}$ are admissible in the sense of Definition 6.1.

If $\mathfrak{D}$ is an admissible operator, then by assumption, for $a, b \in \Gamma_{0}(\zeta)$

$$
\langle\mathfrak{D} a, \mathfrak{D} b\rangle_{2}=\langle a, b\rangle_{\mathfrak{D}}+\langle a, \mathfrak{R} b\rangle_{2},
$$

where $\mathfrak{R} \in \Gamma($ End $\zeta)$ satisfies Property * with respect to the inner product

$$
\langle a, b\rangle_{\mathfrak{D}}=\left\langle\nabla_{A} a, \nabla_{A} b\right\rangle_{2}+\langle q a, q b\rangle_{2} \text {. }
$$


Here, $A$ is a connection on $\zeta$ and $q \in \Gamma(\operatorname{End} \zeta)$. The metric $\langle\cdot, \cdot\rangle_{\mathfrak{D}}$ on $\Gamma_{0}(\zeta)$ defines the Hilbert space $H_{\mathfrak{D}}$ of Definition 6.2. Similarly, one obtains the metric $\langle\cdot, \cdot\rangle_{\mathfrak{D} *}$ on $\Gamma_{0}\left(\zeta^{\prime}\right)$ and the space $H_{\mathfrak{D} *}$ from the adjoint $\mathfrak{D}^{*}$.

For an admissible operator, $\mathfrak{D}$, it is possible to define three indices, $\bar{i}(\mathfrak{D})$ $=\operatorname{dim}\left(\operatorname{ker} \mathfrak{D} \cap H_{\mathfrak{D}}\right)-\operatorname{dim}\left(\operatorname{ker} \mathfrak{D}^{*} \cap L_{2}\right) ; \quad i(\mathfrak{D})=\operatorname{dim}\left(\operatorname{ker} \mathfrak{D} \cap L_{2}\right)-\operatorname{dim}\left(\operatorname{ker} \mathfrak{D}^{*} \cap L_{2}\right)$, and $-\bar{i}\left(\mathfrak{D}^{*}\right)$. Due to Lemma 6.3 , these indices automatically satisfy

$$
\bar{i}(\mathfrak{D}) \geqq i(\mathfrak{D}) \geqq-\bar{i}\left(\mathfrak{D}^{*}\right) .
$$

The above indices are the topic in this section, and the principle result follows (see also Lemma 7.9).

Proposition 7.2. Let $\mathfrak{D}$ be an admissible operator. Then $\mathfrak{D}$ extends to a Fredholm map from $H_{\mathfrak{D}}$ to $L_{2}$ and similarly for $\mathfrak{D}^{*}$. Let $u \in \Gamma\left(\operatorname{Hom}\left(\zeta, \zeta^{\prime}\right)\right)$ be such that $u^{*} u \in \Gamma(\operatorname{End} \zeta)$ and $u u^{*} \in \Gamma\left(\operatorname{End} \zeta^{\prime}\right)$ satisfy Property $*$ for $\langle\cdot, \cdot\rangle_{\mathfrak{D}}$ and $\langle\cdot, \cdot\rangle_{\mathfrak{D} *}$, respectively. Then $\bar{i}(\mathfrak{D}+u)=\bar{i}(\mathfrak{D})$ and $\bar{i}\left(\mathfrak{D}^{*}+u\right)=\bar{i}\left(\mathfrak{D}^{*}\right)$.

The reader may compare Proposition 7.2 (and Lemma 7.9) with the weighted Sobolev space theorems of [24] and [25]. In the Yang-Mills context, see the $L_{2}$ results in [26].

The first relevant example comes from the Yang-Mills theory on $\mathbb{R}^{4}$ :

Lemma 7.3. Let $A \in \mathfrak{A}$ on $\mathbb{R}^{4}$ and let $\delta_{A}=\left(\sqrt{2} P_{-} D_{A}, D_{A}^{*}\right)$. Then $\delta_{A}$ is admissible and the numbers $\bar{i}\left(\delta_{A}\right)$ and $\bar{i}\left(\delta_{A}^{*}\right)$ and the same for $A$ and $A+a$ if $a \in H_{A}$.

The second relevant example concerns the Yang-Mills-Higgs theory of Sect. 5.

Lemma 7.4. Let $c=(A, \Phi) \in \mathbb{C}$ on $\mathbb{R}^{3}$. Define $\mathfrak{D}_{c}$ as in Definition 5.4. Then $\mathfrak{D}_{c}$ is admissible and the numbers $\bar{i}\left(\mathfrak{D}_{c}\right)$ and $\bar{i}\left(\mathfrak{D}_{c}^{*}\right)$ the same for $c$ and $c+\psi$ if $\psi \in H_{c}$.

Proof of Lemma 7.3, assuming Proposition 7.2. The admissibility of $\delta_{A}$ follows from Lemma 6.7. If $a \in H_{A}$, then $\delta_{A+a}-\delta_{A}=a \circ$ where $a \circ \in \Gamma\left(\operatorname{Hom}\left(\hat{\mathfrak{g}}^{1}, P_{-} \hat{\mathfrak{g}}^{2} \oplus \hat{\mathfrak{g}}^{0}\right)\right)$ is

$$
a \circ v=\left(\sqrt{2} P_{-}(a \wedge v+v \wedge a),-*(a \wedge * v-v \wedge * a)\right) .
$$

This homomorphism is essentially multiplication by $a$. Thus $(a \circ)^{*} a \circ \in \Gamma\left(\operatorname{End}\left(\hat{\mathrm{g}}^{1}\right)\right)$ is basically multiplication by $a^{2}$. Observe that

$$
\left\langle\left(1-\chi_{r}\right) \psi_{1},(a \circ)^{*} a \circ \psi_{2}\right\rangle_{2} \leqq c\left\|\left(1-\chi_{r}\right)|a|^{2}\right\|_{2}\left\|\psi_{1}\right\|_{4}\left\|\psi_{2}\right\|_{4},
$$

where the constant $c$ is independent of $\psi_{1}, \psi_{2} \in \Gamma_{0}\left(\hat{\mathrm{g}}^{1}\right)$ and independent of $r<\infty$. Because $a \in H_{A},|a|^{2} \in L_{2}$ (cf. Lemma 6.3) so Eq. (7.4) and Lemma 6.3 imply that $\left(a^{\circ}\right)^{*} a \circ$ satisfies Property * with respect to $\langle\cdot, \cdot\rangle_{H_{A}}$. One analyzes $(a \circ)\left(a^{\circ}\right)^{*}$ similarly, and one finds that it also satisfies Property ${ }^{*}$ with respect to $\langle\cdot, \cdot\rangle_{H_{A}}$. Thus, Lemma 7.3 is a corollary to Proposition 7.2.

Proof of Lemma 7.4, assuming Proposition 7.2. In this case, admissibility of $\mathfrak{D}_{c}$ follows from Lemma 6.8. If $\psi \in H_{c}$ and $\eta \in \Gamma_{0}\left(\hat{\mathfrak{g}}^{\prime}\right)$, then

$$
\mathfrak{D}_{c+\psi} \eta-\mathfrak{D}_{c} \eta \equiv \psi(\eta)=-\left[\psi^{0}, \eta\right]+\tau^{j}\left[\psi^{j}, \eta\right] .
$$

Let $r<\infty$ be given and let $\beta_{r}(x)=\beta(x / r) \in C_{0}^{\infty}\left(\mathbb{R}^{3}\right)$ where $0 \leqq \beta(x)$ and $\beta(x)=1$ if $|x|<1$ and $\beta(x)=0$ if $|x|>3 / 2$. Let $\eta_{1}, \eta_{2} \in \Gamma_{0}\left(\hat{\mathfrak{g}}^{\prime}\right)$. Then 


$$
\begin{aligned}
\left.K\left(1-\chi_{r}\right) \psi\left(\eta_{1}\right), \psi\left(\eta_{2}\right)\right\rangle_{2} \mid & \leqq\left\|\left(1-\beta_{r / 2}\right) \psi\left(\eta_{1}\right)\right\|_{2}\left\|\left(1-\beta_{r / 2}\right) \psi\left(\eta_{2}\right)\right\|_{2} \\
& \leqq\left\|\left(1-\beta_{r / 2}\right) \psi\right\|_{c}^{2}\left\|\eta_{1}\right\|_{c}\left\|\eta_{2}\right\|_{c} .
\end{aligned}
$$

The last line in Eq. (7.7) follows from Lemma 6.6 of [6]. Using Lemma 6.3 one observes that because $\psi \in H_{c}$,

$$
\lim _{r \rightarrow \infty}\left\|\left(1-\beta_{r / 2}\right) \psi\right\|_{c}^{2} \rightarrow 0 .
$$

Equations (7.7) and (7.8) imply that $\psi^{*} \psi(\cdot) \in \Gamma\left(\right.$ End $\left.\hat{\mathfrak{g}}^{1}\right)$ satisfies Property * of Definition 6.1 with respect to $\langle\cdot, \cdot\rangle_{c}$. The analysis for $\psi \psi^{*}$ is similar. Thus Lemma 7.4 is also a corollary to Proposition 7.2.

The remainder of this section is occupied with proving Proposition 7.2.

Proof of Proposition 7.2. The proof begins with the following observation about the domain of $\mathfrak{D}$.

Lemma 7.5. Let $\mathfrak{D}$ be an admissible operator. It extends to a bounded, Fredholm operator from $\mathrm{H}_{\mathfrak{D}}$ to $L_{2}$.

Proof of Lemma 7.5. Let $\psi_{i} \in \Gamma_{0}(\zeta)$ be a Cauchy sequence in $H_{\mathfrak{D}}$ with limit $\psi$. Due to Eq. (7.2) and Lemma 6.4, the sequence $\left\{\mathfrak{D} \psi_{i}\right\} \subset L_{2}\left(\zeta^{\prime}\right)$ is Cauchy. Then $\mathfrak{D} \psi$ is by definition $\lim \left(\mathfrak{D} \psi_{i}\right) \in L_{2}\left(\zeta^{\prime}\right)$. This extends $\mathfrak{D}$ to $H_{\mathfrak{D}}$.

Consider the statement that $\mathfrak{D}: H_{\mathfrak{D}} \rightarrow L_{2}$ is Fredholm: Let $\overline{\mathfrak{D}}^{*}: L_{2} \rightarrow H_{\mathfrak{D}}$ denote the adjoint of $\mathfrak{D}$. By Lemma 6.6, dim $\operatorname{ker} \mathfrak{D} \cap H_{\mathfrak{D}}<\infty$; because ker $\mathfrak{D}^{*}$

$=\operatorname{ker} \mathfrak{D}^{*} \cap L_{2} \subset \operatorname{ker} \mathfrak{D}^{*} \cap H_{\mathfrak{D}^{*}}, \operatorname{dim} \operatorname{ker} \overline{\mathfrak{D}}^{*}<\infty$, too. If $\operatorname{Ran} \mathfrak{D}$ is closed, then $\mathfrak{D}$ is Fredholm. The closure of Ran $\mathfrak{D}$ is a corollary of the next lemma, cf. [27, Theorem IV.5.2].

Lemma 7.6. If $\mathfrak{D}$ is admissible there exists $\gamma>0$ such that $\|\mathfrak{D} \psi\|_{2}^{2} \geqq \gamma\langle\psi, \psi\rangle_{\mathfrak{D}}$ for such all $\psi \in H_{\mathfrak{D}}$ which are $H_{\mathfrak{D}}$-orthogonal to $\operatorname{ker} \mathfrak{D}$.

Proof of Lemma 7.6. Suppose to the contrary that no such $\gamma$ exists. Then there exists a sequence $\left\{\psi_{i}\right\} \in H_{\mathfrak{D}}$ with

(1) $\left\|\mathfrak{D} \psi_{i}\right\|_{2} \rightarrow 0$

(2) $\left\|\psi_{i}\right\|_{H_{\mathfrak{D}}}=1$,

(3) $\left\langle\psi_{i}, \eta\right\rangle_{H_{\mathfrak{D}}}=0$ for all $\eta \in \operatorname{ker} \mathfrak{D} \cap H_{\mathfrak{D}}$.

Due to Eq. (7.9.2), there exists an $H_{\mathfrak{D}}$-weak, limit, $\psi$ of $\left\{\psi_{i}\right\}$. Lemma 6.3 and the Rellich lemma implies that $\psi \rightarrow \psi_{i}$ strongly in $L_{2}$ on bounded domains. By Eq. (7.9.1), $\mathfrak{D} \psi=0$ and therefore Eq. (7.9.3) implies that $\psi=0$. However, Eq. (6.6) now implies that $\lim \left\langle\psi_{i}, \psi_{i}\right\rangle_{\mathfrak{D}}$ is zero which contradicts Eq. (7.9.2). Hence, $\gamma$ exists as claimed.

As $\mathfrak{D}$ is a Fredholm operator, the index $\bar{i}(\mathfrak{D})=\operatorname{dim}\left(\operatorname{ker} \mathfrak{D} \cap H_{\mathfrak{D}}\right)$ $-\operatorname{dim}\left(\operatorname{ker} \mathfrak{D}^{*} \cap L_{2}\right)$ is well defined. Now consider its behavior under perturbation.

Lemma 7.7. Let $\mathfrak{D}$ be an admissible operator, and let $u \in \Gamma\left(\operatorname{Hom}\left(\zeta, \zeta^{\prime}\right)\right)$ be such that $u^{*} u \in \Gamma($ End $\zeta)$ satisfies Property ${ }^{*}$ with respect to $\langle\cdot, \cdot\rangle_{\mathfrak{D} \cdot}$. Then $\mathfrak{D}+u$ is a Fredholm operator from $H_{\mathfrak{D}}$ to $L_{2}\left(\zeta^{1}\right)$ and

$$
\bar{i}(\mathfrak{D}+u)=\bar{i}(\mathfrak{D})
$$


Proof of Lemma 7.7. First observe that because Property * is satisfied by $u^{*} u$, $\operatorname{Domain}(u) \supset H_{\mathfrak{D}}$. Thus $\mathfrak{D}$ and $\mathfrak{D}+u$ have the same domain. The lemma is proved by showing that $u: H_{\mathfrak{D}} \rightarrow L_{2}\left(\zeta^{\prime}\right)$ is a $\mathfrak{D}$ compact operator, cf. Theorem IV.5.26 of [27]. For this purpose, consider a sequence $\left\{\psi_{i}\right\} \in H_{\mathfrak{D}}$ with both $\left\|\mathfrak{D} \psi_{i}\right\|_{2}$ and $\left\|\psi_{i}\right\|_{H_{\mathcal{D}}}$ bounded. One must show that $u \psi_{i}$ has a strongly convergent subsequence in $L_{2}$. The sequence, $\left\{\psi_{i}\right\}$ has a $H_{\mathfrak{D}}$-weakly convergent subsequence - also denoted $\left\{\psi_{i}\right\}$. Let $\psi$ denote the weak limit and consider

$$
\left\|u\left(\psi_{i}-\psi\right)\right\|_{2}^{2}=\left\langle\psi_{i}-\psi, u^{*} u\left(\psi_{i}-\psi\right)\right\rangle_{2} .
$$

Due to Property *, the right hand side of Eq. (7.10) converges to zero. Therefore, $\left\{u \psi_{i}\right\}$ converges strongly in $L_{2}$ as required.

One can now reverse the roles of $\mathfrak{D}$ and $\mathfrak{D}^{*}$ by considering $\mathfrak{D}^{*}$ as a bounded operator from $H_{\mathfrak{D}^{*}}$ to $L_{2}(\zeta)$. One obtains

Lemma 7.8. Let $\mathfrak{D}$ be an admissible operator. Let $u \in \Gamma\left(\operatorname{Hom}\left(\zeta, \zeta^{\prime}\right)\right)$ be such that $u u^{*} \in \Gamma\left(\right.$ End $\left.\zeta^{\prime}\right)$ satisfies Property $*$ with respect to $\langle\cdot, \cdot\rangle_{\mathfrak{D}^{*}}$. Then $\mathfrak{D}^{*}+u^{*}$ is a Fredholm operator from $\mathrm{H}_{\mathfrak{D}^{*}}$ to $L_{2}(\zeta)$ and

$$
\bar{i}\left(\mathfrak{D}^{*}+u^{*}\right)=\bar{i}\left(\mathfrak{D}^{*}\right) \text {. }
$$

Proposition 7.2 follows directly from Lemmas 7.5, 7.7, and 7.8.

Concerning the index $i(\mathfrak{D})$, one has

Lemma 7.9. Let $\mathfrak{D}$ be an admissible operator. Let $\alpha<\frac{1}{4}(n-2)$. If $\psi \in \operatorname{ker} \mathfrak{D} \cap H_{\mathfrak{D}}$, then $\left(1+|x|^{2}\right)^{\alpha} \psi \in H_{\mathfrak{D}}$. In particular, if $n>4$, then $\operatorname{ker} \mathfrak{D} \cap H_{\mathfrak{D}}=\operatorname{ker} \mathfrak{D} \cap L_{2}$ and $\bar{i}(\mathfrak{D})$ $=i(\mathfrak{D})=-\bar{i}\left(\mathfrak{D}^{*}\right)$.

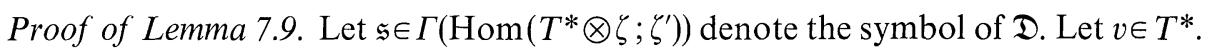
As a consequence of Eqs. (7.2) and (7.3),

$$
\mathfrak{s}^{*}(v) \mathfrak{s}(v)=|v|^{2} .
$$

Now consider for $R>0$

$$
\psi_{\alpha, R}=\left\{\begin{array}{lll}
\left(1+|x|^{2}\right)^{\alpha} \psi & \text { for } & |x|<R \\
\left(1+R^{2}\right)^{\alpha} \psi & \text { for } & |x|>R
\end{array}\right.
$$

Then $\psi_{\alpha, R} \in H_{\mathfrak{D}}$ and

$$
\mathfrak{D} \psi_{\alpha, R}=\left\{\begin{array}{lll}
\alpha\left(1+|x|^{2}\right)^{-1} \mathfrak{s}\left(d|x|^{2}\right) \psi_{\alpha, R} & \text { for } & |x|<R, \\
0 & \text { for } & |x|>R .
\end{array}\right.
$$

Squaring both sides of Eq. (7.13) one obtains

$$
\left\|\mathfrak{D} \psi_{\alpha, \boldsymbol{R}}\right\|_{2}^{2} \leqq 4 \alpha^{2}\left\|\left(1+|x|^{2}\right)^{-1 / 2} \psi_{\alpha, \boldsymbol{R}}\right\|_{2}^{2} \text {. }
$$

With Eqs. (7.2), (7.3), and (6.6) one deduces from Eq. (7.14) the following inequality which is valid for any $\varepsilon>0$ :

$$
(1-\varepsilon)\left\|\psi_{\alpha, R}\right\|_{\mathcal{D}} \leqq 2 \alpha\left\|\left(1+|x|^{2}\right)^{-1 / 2} \psi_{\alpha, R}\right\|_{2}+c(\varepsilon)\|\psi\|_{\mathcal{D}} .
$$

Now apply Lemma 6.3. The result is

$$
\left((1-\varepsilon)-\frac{4 \alpha}{n-2}\right)\left\|\psi_{\alpha, R}\right\|_{\mathfrak{D}} \leqq c(\varepsilon)\|\psi\|_{\mathfrak{D}} .
$$


Observe that for $\alpha<\frac{1}{4}(n-2)$, Eq. (7.16) produces an $R$-independent bound for $\left\|\psi_{\alpha, R}\right\|_{\mathfrak{D}}$ and for such $\alpha, \psi_{\alpha} \in H_{\mathfrak{D}}$. The concluding statement of the lemma comes from Lemma 6.3.

\section{The Index Theorem for Yang-Mills}

The subject here is the operator

$$
\delta_{A}=\left(\sqrt{2} P_{-} D_{A}, D_{A}^{*}\right): \Gamma\left(\hat{\mathfrak{g}}^{1}\right) \rightarrow \Gamma\left(P_{-} \hat{\mathfrak{g}}^{2} \oplus \hat{\mathfrak{g}}^{0}\right),
$$

and the number $\operatorname{dim} \operatorname{ker} \delta_{A}-\operatorname{dim} \operatorname{ker} \delta_{A}^{*}$. As before, $\delta_{A}^{*}$ is the formal $L_{2}$-adjoint of $\delta_{A}$. The proofs of Proposition 4.2 and Lemma 4.5 result from the study of $\delta_{A}$. Proposition 4.2 is a consequence of the proposition below.

Proposition 8.1. Let $A \in \mathfrak{A}$. Define $p_{1}(A)$ by Eq. (4.1) and define $\delta_{A}$ and $\delta_{A}^{*}$ as above. Then

$$
\bar{i}\left(\delta_{A}\right)=i\left(\delta_{A}\right)=-\bar{i}\left(\delta_{A}^{*}\right)=p_{1}(A) .
$$

Proof of Proposition 4.2 assuming Proposition 8.1. The first assertion is a consequence of Lemma 7.3, and the second is directly restated in Proposition 8.1. Only the third assertion of Proposition 4.2 need be discussed. To prove this assertion, it is sufficient to establish that $\operatorname{dim}\left(\operatorname{ker} \delta_{A}^{*} \cap L_{2}\right) \geqq 8$ when $A \in \mathfrak{A}$ is a solution to the Yang-Mills equations. To begin, note that the vector bundle $P_{-} \wedge T^{*} \oplus \mathbb{R}$ admits a covariantly constant, isometric action of the unit quaternions. In fact, $P_{-} \hat{2}_{2} T^{*} \oplus \mathbb{R} \stackrel{\sim}{\rightarrow} \mathbb{R}^{4} \times \mathbb{H}$; this is obtained by sending

$$
(u, \phi) \mapsto \psi=u^{k} \omega^{k}+\phi,
$$

where $\left\{\omega^{k}\right\}_{k=1}^{3}$ are a covariantly constant, orthonormal basis for $P_{-} \hat{2} T^{*}$. As a consequence, $P_{-} \hat{\mathfrak{g}}^{2} \oplus \hat{\mathfrak{g}}^{0}$ admits an isometric action of the unit quaternions by right multiplication:

$$
\omega^{i} \cdot \psi=-u^{i}-\varepsilon^{i j k} u^{j} \omega^{k}+\phi \omega^{i}
$$

In this notation, one obtains the following expressions for $\delta_{A}^{*} \psi, \psi \in \Gamma\left(P_{-} \hat{\mathfrak{g}}^{2} \oplus \hat{\mathfrak{g}}^{0}\right)$ :

$$
\delta_{A}^{*} \psi=D_{A} \phi+\omega^{k}\left(D_{A} u^{k}\right)
$$

where $\left\{\omega^{k}\right\}$ acts on $\Gamma\left(\hat{\mathrm{g}}^{1}\right)$ as in Eq. (3.1). One can readily compute that

$$
\omega^{k} \cdot\left(\delta_{A}^{*} \psi\right)=\delta_{A}^{*} \omega^{k} \cdot \psi \text {. }
$$

Observe that Eq. (8.2) implies that $\operatorname{ker} \delta_{A}^{*}$ also admits an isometric action of the quaternions. Therefore, $\operatorname{dim} \operatorname{ker} \delta_{A}^{*}$ is an integer multiple of 4.

Now suppose that $A$ is a solution to the Yang-Mills equations on $\mathbb{R}^{4}$ with finite action. In particular, $P_{-} F_{A}$ satisfies $D_{A}^{*} P_{-} F_{A}=0$. Thus $\left(P_{-} F_{A}, 0\right) \in \operatorname{ker} \delta_{A}^{*}$ and so $\operatorname{dim} \operatorname{ker} \delta_{A}^{*} \geqq 4$. In fact, $\operatorname{dim} \operatorname{ker} \delta_{A}^{*} \geqq 8$. To see this, one can consider

$$
\left.\psi=\left(x^{4} F_{-}^{k}+\varepsilon^{i j k} x^{i} F_{-}^{j}\right) \omega^{k},-x^{i} F_{-}^{i}\right) .
$$


Here, $F_{-}^{k}=\left(\omega^{k}, P_{-} F_{A}\right)$ and $\left\{x^{v}\right\}_{v=1}^{4}$ are Cartesian coordinates on $\mathbb{R}^{4}$ which are defined by the requirement that

$$
\omega^{i}\left(d x^{4}\right)=-d x^{i}
$$

Notice that

$$
\psi=\left(x^{4}+x^{i} \omega^{i}\right) \cdot F_{-}^{k} \omega^{k} .
$$

If $A$ is the pull back by inverse stereographic projection of a connection which is smooth on $S^{4}$, then

$$
\left|F_{A}\right|(x) \leqq \text { const }_{A}\left(1+|x|^{2}\right)^{2}
$$

In particular, Eq. (8.6) is satisfied by every finite action solution of the $\mathbb{R}^{4}$-YangMills equations [28] and as a consequence, $\psi \in L_{2}\left(P_{-} \hat{\mathfrak{g}}^{2} \oplus \hat{\mathfrak{g}}^{0}\right)$.

Lemma 8.2. Define $\psi$ by Eq. (8.6). Then $\delta_{A}^{*} \psi=0$.

Proof of Lemma 8.2. Using Eqs. (8.1), (8.2), and (8.5), one obtains the sequence below:

$$
\begin{aligned}
\delta_{A}^{*} \psi & =\omega^{k}\left(D_{A}\left(x^{4} F_{-}^{k}\right)\right)+\omega^{i}\left(\omega^{k}\left(D_{A}\left(x^{i} F_{-}^{k}\right)\right)\right. \\
& =\omega^{k}\left(d x^{4}\right) F_{-}^{k}-d x^{k} F_{-}^{k}-\varepsilon^{i k j} \omega^{j}\left(d x^{i}\right) F_{-}^{k} \\
& =-2 d x^{k} F_{-}^{k}+\varepsilon^{i k j} \omega^{j}\left(\omega^{i}\left(d x^{4}\right)\right) F_{-}^{k} \\
& =-2 d x^{k} F_{-}^{k}+\varepsilon^{i j k} \varepsilon^{i j l} d x^{l} F_{-}^{k}=0,
\end{aligned}
$$

which is the desired result.

Clearly, $\psi \neq h \cdot F_{-}^{k} \omega^{k}$ for any constant $h \in \mathbb{H}$. Thus, $\operatorname{dim} \operatorname{ker} \delta_{A}^{*} \geqq 8$, and due to Proposition 8.1, $\operatorname{dim} \operatorname{ker} \delta_{A} \geqq p_{1}(A)+8$ as claimed. This establishes Proposition 4.2.

The remainder of this section contains the proof of Proposition 8.1. Lemma 4.5 is proved at the end of this section.

To begin, recall that Lemma 7.3 states that $\delta_{A}$ and $\delta_{A}^{*}$ are admissible operators, so Proposition 7.2 is applicable. The principal tool for computing the indices is Corollary 2.2 of Uhlenbeck [18] which is reworded slightly as Proposition 4.3. An immediate corollary to Proposition 4.3 is

Proposition 8.3. Let $A \in \mathfrak{U}$. There exists a connection $\hat{A} \in \mathfrak{U}$ and $\eta \in C^{\infty}\left(\mathbb{R}^{4} ; G\right)$ with the following properties:

(1) As a bundle with connection, $\left(\mathbb{R}^{4} \times G, \hat{A}\right)$ is isomorphic to the pull-back via inverse stereographic projection of a pair $\left(P^{\prime}, A^{\prime}\right)$, where $P^{\prime} \rightarrow S^{4}$ is a principal $G$-bundle, and $A^{\prime}$ is a smooth connection on $P^{\prime}$.

(2) $\hat{A}-\left(\eta A \eta^{-1}+\eta d \eta^{-1}\right) \in H_{\hat{A}}$.

Proposition 8.1 is used in the following way: Because $\operatorname{dim}\left(\operatorname{ker} \delta_{A} \cap H_{A}\right)$ and $\operatorname{dim}\left(\operatorname{ker} \delta_{A}^{*} \cap H_{A}\right)$ are Aut $\left(\mathbb{R}^{4} \times G\right)$ invariant, Lemma 7.3 and Propositions 4.4 and 8.3 imply that $\bar{i}\left(\delta_{A}\right)$ is the same as $\bar{i}\left(\delta_{\hat{A}}\right)$ where $\hat{A} \in \mathfrak{U}$ is a self-dual connection on $\mathbb{R}^{4}$ with the property that $p_{1}(\hat{A})=p_{1}(A)$.

If $A \in \mathfrak{A}$ is a self-dual connection, then for all $\psi \in H_{A},\left\|\delta_{A}^{*} \psi\right\|_{2}=\left\|\nabla_{A} \psi\right\|_{2}$ and so $\operatorname{ker}\left(\delta_{A}^{*} \cap H_{A}\right)=0 . \operatorname{Dim} \operatorname{ker} \delta_{A}$ is described in the next lemma.

Lemma 8.4. Let $A \in \mathfrak{U}$ be self-dual. Then $\operatorname{dim}\left(\operatorname{ker} \delta_{A} \cap H_{A}\right)=\operatorname{dim}\left(\operatorname{ker} \delta_{A} \cap L_{2}\right)$ $=p_{1}(A)$. 
Proof of Proposition 8.1, assuming Lemma 8.4. Let $A$ be the connection in question. Due to Lemma 8.4, and Propositions 7.2 and 8.3, $\bar{i}\left(\delta_{A}\right)=p_{1}(A)=-\bar{i}\left(\delta_{A}^{*}\right)$ for the operator $\delta_{A}$.

Proof of Lemma 8.4. Consider a self-dual connection $A^{\prime}$ on a principal $G$-bundle $P^{\prime} \rightarrow S^{4}$. There exists the following exact sequence on $S^{4}$ :

$$
0 \rightarrow \Gamma\left(\tilde{\mathfrak{g}}^{0}\right) \stackrel{D_{A^{\prime}}}{\longrightarrow} \Gamma\left(\tilde{\mathfrak{g}}^{1}\right) \stackrel{P_{-} D_{A^{\prime}}}{\longrightarrow} \Gamma\left(P_{-} \tilde{\mathfrak{g}}^{2}\right) \longrightarrow 0 .
$$

Here, in order to avoid confusion between bundles on $S^{4}$ and $\mathbb{R}^{4}, \tilde{\mathfrak{g}}^{p}=\left(P^{\prime} \times{ }_{\text {Ad }} \mathfrak{g}\right)$ $\otimes \underset{p}{\wedge} T^{*}\left(S^{4}\right)$. The cohomology of this sequence was computed in [17]: As $A^{\prime}$ is self-dual, $h^{2}=0$ and $h^{1}-h^{0}=p_{1}(\tilde{\mathfrak{g}})-\operatorname{dim} G$. Of course, if $A^{\prime}$ is irreducible, then $h^{0}=0$ and it is always true that $h^{0} \leqq \operatorname{dim} G$. The cohomology in dimension 1 can be represented by elements $\left\{a_{i}^{\prime}, i \in\left(1, \ldots, h^{1}\right)\right\} \in \Gamma\left(\tilde{\mathfrak{g}}^{1}\right)$ with $a_{i}^{\prime}$ (south pole) $=0$.

When pulled back to $\mathbb{R}^{4}$ by stereographic projection, $\mathfrak{s}^{-1}\left(P^{\prime}, A^{\prime},\left\{a_{i}^{\prime}\right\}\right)$ is isomorphic to data $\left(\mathbb{R}^{4} \times G, A,\left\{a_{i}\right\}\right)$. The $a_{i}$ satisfy $P_{-} D_{A} a_{i}=0$, and $a_{i} \in H_{A}\left(\hat{\mathrm{g}}^{1}\right) \cap L_{2}\left(\hat{\mathrm{g}}^{1}\right)$. Indeed, as $a_{i}^{\prime}($ south pole $)=0$,

$$
\left|a_{i}\right| \leqq \text { const } \cdot\left(1+|x|^{2}\right)^{-3 / 2} \text {. }
$$

Unfortunately, $a_{i} \notin \operatorname{ker} \delta_{A}$, necessarily. However, according to Lemma 7.5, $\operatorname{Im} \delta_{A}^{*} \subset L_{2}$ is a closed, linear subspace of finite codimension. Consider the projection of $\operatorname{Span}\left\{a_{i}\right\}$ onto $\left(\operatorname{Im} \delta_{A}^{*}\right)^{\perp}$, and denote it $\Pi\left\{a_{i}\right\}$.

Lemma 8.5. Let $\Pi\left\{a_{i}\right\}$ be defined as in the preceding paragraph. Then $\operatorname{dim} \Pi\left\{a_{i}\right\}$ $=\operatorname{dim} \operatorname{Span}\left\{a_{i}^{\prime}\right\}=h^{1}$.

Proof of Lemma 8.5. Elements of $\Pi\left\{a_{i}\right\}$ pull back via stereographic projection to $L_{1,2}$ sections of $\tilde{\mathfrak{g}}^{1}$ which will be denoted $\left\{\Pi a_{i}^{\prime}\right\}$. For each $i$,

$$
a_{i}^{\prime}-\Pi\left(a_{i}^{\prime}\right) \in \operatorname{Im}\left(D_{A^{\prime}}: L_{2,2}\left(\tilde{\mathfrak{g}}^{0}\right) \rightarrow L_{1,2}\left(\tilde{\mathfrak{g}}^{1}\right)\right) .
$$

As the $L_{1,2}$ cohomology and the smooth cohomology of the complex in Eq. (8.8) agree, $\left\{\Pi a_{i}^{\prime}\right\}$ must generate $H^{1}$ of that complex. Hence, $\operatorname{dim} \Pi\left\{a_{i}\right\}=\operatorname{dim}\left\{a_{i}^{\prime}\right\}=h^{1}$ as claimed.

One obtains $\operatorname{dim} G-h^{0}$ additional linearly independent elements in $\operatorname{ker} \delta_{A} \cap L_{2}$ with the following construction. Return to $S^{4}$ and choose a basis $\left\{\sigma_{\alpha}\right\}_{\alpha=1}^{\operatorname{dim} G}$ for $\left.\tilde{\mathfrak{g}}^{0}\right|_{s}$, where $s=$ south pole. By parallel transport with $A^{\prime}$ along the great circles through $s$, extend each $\sigma_{\alpha}$ to a section $\phi_{\alpha}^{\prime}$ of $\tilde{\mathfrak{g}}^{0}$ over the southern hemisphere. By smoothly interpolating each $\phi_{\alpha}^{\prime}$ to zero in the northern hemisphere, one obtains smooth sections $\phi_{\alpha} \in \Gamma\left(\tilde{\mathfrak{g}}^{0}\right)$ which satisfy

$$
\left.\phi_{\alpha}^{\prime}\right|_{s}=\sigma_{\alpha}, \quad \text { and }\left.\quad D_{A^{\prime}} \phi_{\alpha}^{\prime}\right|_{s}=0 .
$$

Now pull back the data $\left(P^{\prime}, A^{\prime}, \phi_{\alpha}^{\prime}\right)$ to $\mathbb{R}^{4}$. The data is isomorphic to $\left(P, A, \phi_{\alpha}\right)$ and $\phi_{\alpha}$ satisfies

$$
\lim _{|x| \rightarrow \infty}\left|\phi_{\alpha}\right|=1, \quad \lim _{|x| \rightarrow \infty}\left(\phi_{\alpha}, \phi_{\beta}\right)=\delta_{\alpha \beta}, \quad \text { and } \quad D_{A} \phi_{\alpha} \in H_{A} .
$$

In addition, as $A$ is self-dual, $P_{-} D_{A} D_{A} \phi_{\alpha}=0$. Observe that the projection of $\left\{D_{A} \phi_{\alpha}\right\}$ orthogonal to $\operatorname{Im} \delta_{A}^{*} \subset L_{2}\left(\hat{\mathrm{g}}^{1}\right)$ is necessarily $\operatorname{dim} G-h^{0}$ dimensional. This is because $\phi_{\alpha} \notin H_{A}$. This projection is also linearly independent of $\Pi\left\{a_{i}\right\}$; if not, one 
would obtain a contradiction by pulling back to $S^{4}$ as in the proof of Lemma 8.5. Thus, $\operatorname{dim}\left(\operatorname{ker} \delta_{A} \cap L_{2}\right) \geqq p_{1}(A)$.

By reversing this last argument, one shows that $\operatorname{dim}\left(\operatorname{ker} \delta_{A} \cap H_{A}\right)$ $\left(=\operatorname{dim} \operatorname{ker} \delta_{A} \cap L_{2}\right) \leqq p_{1}(A)$. This will establish Lemma 8.4. Indeed, assume now that there exists $a \in \operatorname{ker}\left(\delta_{A} \cap H_{A}\right)$ which is linearly independent from $\Pi\left\{a_{i}, D_{A} \phi_{\alpha}\right\}$. Then pulling $a$ back to $S^{4}$ via the stereographic projection yields $a^{\prime} \in L_{1,2}\left(\tilde{\mathfrak{g}}^{1}\right)$ which satisfies (on $S^{4}$ )

$$
P_{-} D_{A^{\prime}} a^{\prime}=0
$$

and therefore $a^{\prime}=D_{A^{\prime}} \tilde{\psi}$ for some $\tilde{\psi} \in L_{2,2}\left(\tilde{\mathfrak{g}}^{0}\right)$. But this implies that on $\mathbb{R}^{4}, a=D_{A} \psi$ for some $\psi \in \Gamma\left(\mathbb{R}^{4} \times \mathfrak{g}\right)$. Lemma 4.5 , which is still unproved, forbids this occurrence unless $\psi=0$ and $a=0$.

Proof of Lemma 4.5. Let $\psi \in K$. Then because $D_{A} \psi \in V_{A}$, one concludes from Lemma 7.9 that for any $\varepsilon>0$,

$$
\left(1+|x|^{2}\right)^{-\varepsilon} D_{A} \psi \in L_{2} .
$$

Now consider the pull back of $(A, \psi)$ by stereographic projection to $S^{4},\left(A^{\prime}, \tilde{\psi}\right)$. By assumption, $A^{\prime}$ is smooth, and $\tilde{\psi}$ is smooth on $S^{4} \backslash$ \{south pole\}. Let $\left\{y^{v}\right\}_{v=1}^{4}$ be stereographic coordinates on $S^{4}$ centered at the south pole. In these coordinates,

$$
\left|\delta_{A^{\prime}} D_{A^{\prime}} \tilde{\psi}\right|(y) \leqq \text { const }|y|^{-1}\left|D_{A^{\prime}} \psi^{\prime}\right|(y) .
$$

Due to Eq. (8.13), the right hand side of Eq. (8.14) is in $L_{3}\left(S^{4}\right)$. By elliptic regularity for $\delta_{A^{\prime}}, D_{A^{\prime}} \tilde{\psi} \in L_{1,3}\left(\tilde{\mathfrak{g}}^{1}\right)$ on $S^{4}$ and therefore $\tilde{\psi} \in C^{0}\left(\mathrm{~g}^{0}\right)$ on $S^{4}$ by the Sobolev lemma. If $\operatorname{dim} K>\operatorname{dim} g=\operatorname{dim} G$, there would exist a nonzero $\psi \in K$ with $\tilde{\psi}$ (south pole) $=0$; equivalently, $\lim _{|x| \rightarrow \infty}|\psi|(x) \rightarrow 0$. But $D_{A} * D_{A} \psi=0$ on $\mathbb{R}^{4}$, so $|\psi|$ is subharmonic hence zero everywhere. This is a contradiction. Therefore, $\operatorname{dim} K \leqq \operatorname{dim} G$ as claimed.

\section{Index Theorem for Yang-Mills-Higgs}

Here the subject is the operator $\mathfrak{D}_{c}$, for $c \in \mathbb{C}$ as defined in Sect. 5. Of particular concern are the indices $\bar{i}\left(\mathfrak{D}_{c}\right)$ and $\bar{i}\left(\mathfrak{D}_{c}^{*}\right)$, where $\mathfrak{D}_{c}^{*}$ is the formal $L_{2}$-adjoint of $\mathfrak{D}_{c}$. A proof of Proposition 5.5 results from the calculation of $\bar{i}$. A non-rigorous calculation of $\bar{i}$ has been given by Weinberg [12]. Weinberg's strategy is quite different from the one below.

Proposition 9.1. Let $c \in \mathfrak{C}_{n}, n \in \mathbb{Z}$. Let $\mathfrak{D}_{c}$ be given by Eq. (5.8), and let $\mathfrak{D}_{c}^{*}$ be its formal $L_{2}$-adjoint. Then

$$
\bar{i}\left(\mathfrak{D}_{c}\right)=-\bar{i}\left(\mathfrak{D}_{c}^{*}\right)=4 n .
$$

Proof of Proposition 5.5, assuming Proposition 9.1. Both $\mathfrak{D}_{c}$ and $\mathfrak{D}_{c}^{*}$ commute with right multiplication by $\mathbb{H}$ on $\Gamma\left(\hat{\mathfrak{g}}^{\prime}\right)$. Because of this, one need only show that $\operatorname{ker} \mathfrak{D}_{c}^{*} \cap H_{c} \neq \emptyset$ when $c=(A, \Phi) \in \mathfrak{C}_{n}(n \geqq 0)$ is a solution to Eq. (5.3) which is not a solution to Eq. (5.4). Consider $\psi=* F_{A}-D_{A} \Phi$ as a section of $\hat{\mathfrak{g}}^{\prime}$. The Bianchi identities together with Eq. (5.3) imply that $\mathfrak{D}_{c}^{*} \psi=0$. Proposition 5.5 now follows. 
Proof of Proposition 9.1. Because $\mathfrak{D}_{c}$ is admissible (Lemma 7.4), the kernels in question are finite dimensional. The difference in the dimensions of the kernels of $\mathfrak{D}_{c}$ and $\mathfrak{D}_{c}^{*}$ will be computed with the aid of Lemma 7.4. The first step in the calculation is to prove the following proposition.

Proposition 9.2. Let $c, c^{\prime} \in \mathfrak{C}_{n}, \quad$ for $n \in \mathbb{Z}$. There exists $g \in \mathfrak{G}=\left\{g \in C^{\infty}\left(\mathbb{R}^{3}\right.\right.$; $\mathrm{SU}(2)): g(0)=1\}$ such that $\psi=g c^{\prime}-c \in H_{c}\left(\hat{\mathfrak{g}}^{\prime}\right)$.

A corollary to Lemma 7.4 and Proposition 9.2 is

Corollary 9.3. For $c \in \mathbb{C}$, the integers $\bar{i}\left(\mathfrak{D}_{c}\right)$ and $\bar{i}\left(\mathfrak{D}_{c}^{*}\right)$ are constant on the path components, $\left\{\mathfrak{C}_{n}\right\}_{n \in \mathbb{Z}}$ of $\mathfrak{C}$.

The second step in the calculation of $i$ is to find a convenient $c \in \mathbb{C}_{n}$ to count the zero modes of $\mathfrak{D}_{c}$ and $\mathfrak{D}_{c}^{*}$.

Proposition 9.4. Let $0 \leqq n \in \mathbb{Z}$. There exists $c \in \mathfrak{C}_{n}$ for which $\operatorname{ker} \mathfrak{D}_{c}^{*} \cap H_{c}=\emptyset$ and

$$
\operatorname{ker}\left(\mathfrak{D}_{c} \cap H_{c}\right)=\operatorname{ker}\left(\mathfrak{D}_{c} \cap L_{2}\right)=4 n .
$$

The remainder of this section contains the proofs of Propositions 9.2 and 9.4 .

Proof of Proposition 9.2. The argument is simplified somewhat by the observation that if $c-c^{\prime} \in H_{c}$ then $H_{c}$ and $H_{c^{\prime}}$ are isomorphic Hilbert spaces (this follows using Lemma 6.6 of [6]).

To begin, for $n \in \mathbb{Z}$, let $M_{n}\left(S^{2} ; S^{2}\right)$ denote the space of $C^{\infty}$ maps from $S^{2}$ to $S^{2}$ whose degree is $n$. Equation (3.3) of [6, Sect. 3] provides a map $I: M_{n}\left(S^{2} ; S^{2}\right) \rightarrow \mathbb{C}_{n}$ :

$$
I(e)=(1-\beta(x))(-[e(x /|x|), d e(x /|x|)], e(x /|x|)),
$$

where $e(x /|x|): S^{2} \rightarrow\{\sigma \in \mathfrak{s u}(2):|\sigma|=1\}=S^{2}$, and $\beta(x) \in C_{0}^{\infty}\left(\mathbb{R}^{3}\right)$ equals 1 when $|x| \leqq 1$.

Lemma 9.5. Proposition 9.2 is true if $c, c^{\prime} \in \operatorname{Image}(I)$.

Proof of Lemma 9.5. If $e, e^{\prime} \in M_{n}\left(S^{2} ; S^{2}\right)$ there exists $h \in C^{\infty}\left(S^{2} ; \mathrm{SU}(2)\right)$ such that $e=h e^{\prime} h^{-1}$. Because $C^{\infty}\left(S^{2} ; \mathrm{SU}(2)\right)$ is path connected, there exists $g \in(\mathfrak{5}$ which is equal to $h(x /|x|)$ for $|x|>1$. For $R>0$, let $\beta_{R}(x)=\beta(x / R)$. Then

$$
I(e)-g I\left(e^{\prime}\right)=-\left(1-\beta_{2}\right) e\left(e, g d g^{-1}\right)+\beta_{2}\left(I(e)-g I\left(e^{\prime}\right)\right) .
$$

The lemma now follows from the observations that

$$
|d e|,|d g| \leqq \operatorname{const}\left(1+|x|^{2}\right)^{-1 / 2} \text {, }
$$

and

$$
\left|\nabla_{0} d e\right|,\left|\nabla_{0} d g\right| \leqq \operatorname{const}\left(1+|x|^{2}\right)^{-1} .
$$

One must now consider arbitrary $c=(A, \Phi) \in \mathfrak{C}_{n}$.

Lemma 9.6. Let $c=(A, \Phi) \in \mathfrak{C}_{n}$. There exists $c_{1}=\left(A, \Phi_{1}\right) \in \mathfrak{C}_{n}$ with the properties: (1) $c-c_{1} \in H_{c_{1}}$, and (2) $\nabla_{A} \Phi_{1} \in H_{c_{1}} \cap L_{2}$.

Proof of Lemma 9.6. Observe first that $d(1-|\Phi|) \in L_{2}$ (Lemma 4.12 of [6]) and as a consequence there exists $R<\infty$ so that $\left(A,\left(1-\beta_{R}(1-|\Phi|)\right) \Phi /|\Phi|\right)$ is in $H_{c}$. For this reason there is no loss of generality in assuming that $|\Phi|(x)=1$ if $|x| \geqq 1$. By 
mimicking the proof of Proposition 4.8 of [6], one obtains a unique $\eta \in H_{c}$ satisfying

(1) $\nabla_{A}^{2}(\Phi+\eta)+[\Phi,[\Phi, \eta]]=0$,

(2) $|\Phi+\eta| \leqq 1$,

(3) $(A, \Phi+\eta) \in \mathfrak{C}$.

Let $\Phi_{1}=\Phi+\eta$. From the fact that $\eta \in H_{c}$, one obtains from Eq. (9.4.1) that $\nabla_{A}^{2} \Phi_{1} \in L_{2}\left(\hat{\mathrm{g}}^{0}\right)$. Statement (2) of Lemma 9.6 follows from the a priori estimate of Theorem V.8.1 in [5].

Since $\left(A, \Phi_{1}\right) \in \mathbb{C}$, there exists $R<\infty$ such that $\left|\Phi_{1}\right|(x)>0$ when $|x| \geqq R$. Let $c_{2}=\left(A, \Phi_{2}\right)=\left(A,\left[1-\beta_{R}\left(1-\left|\Phi_{1}\right|\right)\right] \Phi_{1} /\left|\Phi_{1}\right|\right)$. As before, $\left(A, \Phi_{1}\right)-\left(A, \Phi_{2}\right) \in H_{c}$, and one can readily check that $\nabla_{A} \Phi_{2} \in H_{c_{2}} \cap L_{2}$. This implies that $\nabla_{A} \Phi_{2} \in L_{p}\left(\hat{\mathrm{g}}^{1}\right)$ for $p \in[2, \ldots, 6]$.

Lemma 9.7. Let $\left(A, \Phi_{2}\right) \in \mathfrak{C}_{n}$ be as specified above. There exists $e \in M_{n}\left(S^{2} ; S^{2}\right)$ and $g_{1} \in \mathfrak{G}$ such that

$$
\left(g_{1} \Phi_{2} g_{1}^{-1}\right)(x)=e(x /|x|) \quad \text { for } \quad|x| \geqq R .
$$

Proof of Lemma 9.7. Take $e$ to be $\left.\Phi_{2}\right|_{|x|=R}$. The existence of $g_{1}$ follows from the fact that the fibration $0 \rightarrow S^{1} \rightarrow \mathrm{SU}(2) \rightarrow S^{2} \rightarrow 0$ has the homotopy lifting property.

Let $c_{3}=\left(A_{3}, \Phi_{3}\right)=g_{1}\left(A, \Phi_{2}\right)$. Keep in mind that $\nabla_{A_{3}} \Phi_{3} \in\left(H_{c_{3}} \cap L_{2}\right)\left(\hat{\mathfrak{g}}^{\prime}\right)$ and that $\Phi_{3}(x)=e(x /|x|)$ when $|x| \geqq R$.

Lemma 9.8. Let $c_{3}=\left(A_{3}, \Phi_{3}\right)$ be as defined above. There exists $g_{2} \in \mathbb{G}$ such that $c_{4} \equiv\left(A_{4}, \Phi_{4}\right)=g_{2} c_{3}$ satisfies

(1) $\Phi_{4}=\Phi_{3}$,

(2) $A_{4}+\left(1-\beta_{R}\right)[e, d e] \in H_{c_{4}}$.

Proof of Lemma 9.8. Let $A_{3}^{T}=\left(1-\beta_{R}\right)\left[e,\left[A_{3}, e\right]\right]$. As

$$
\nabla_{A_{3}} \Phi_{3}=d e+\left[A_{3}, e\right] \text { for }|x| \geqq R,
$$

one can conclude that $A_{3}^{T} \in L_{4}\left(\hat{\mathfrak{g}}^{1}\right)$. Let $a_{3}^{L}=\left(1-\beta_{R}\right)\left(e, A_{3}\right)$. Observe that for $|x| \geqq 2 R$,

$$
\left(e, F_{A_{3}}\right)=d a_{3}^{L}+\frac{1}{2} \operatorname{trace}_{\mathfrak{s u}(2)}\left(d e \wedge A_{3}^{T}\right)+\left(e, A_{3}^{T} \wedge A_{3}^{T}\right) .
$$

Therefore, $d a_{3}^{L} \in L_{2}\left(\hat{\imath}^{*} T^{*}\right)$. According to Proposition 7.6 of [6], (see also [29]) there exists $a^{\prime} \in \Gamma\left(T^{*}\right) \cap L_{6}\left(T^{*}\right)$ with the properties

(1) $d a^{\prime}=d a_{3}^{L}$,

(2) $\nabla_{0} a^{\prime} \in L_{2}\left(T^{*}\right)$.

One concludes from Eq. (9.7.1) that

$$
a^{\prime}-a_{3}^{L}=d \Lambda
$$

for some $\Lambda \in C^{\infty}\left(\mathbb{R}^{3}\right)$. Let

$$
g_{2}=\cos \left[\left(1-\beta_{R}\right) \frac{\Lambda}{2}\right]+e \sin \left[\left(1-\beta_{R}\right) \frac{\Lambda}{2}\right] .
$$


Define $A_{4}$ to be $g A_{3}$ and then define $A_{4}^{T}$ and $a_{4}^{L}$ appropriately. By construction

(1) $\Phi_{4}=\Phi_{3}$,

(2) $a_{4}^{L}=a^{\prime}$ for $|x|>2 R$, and

(3) $\nabla_{A_{4}} \Phi_{4}=d e+\left[A_{4}, e\right]$ for $|x|>2 R$.

Due to Eq. (9.9.3), and Lemmas 9.6 and 9.7,

$$
A_{4}^{T}+\left(1-\beta_{R}\right)[e, d e] \in H_{c_{4}} \cap L_{2} .
$$

Due to Eqs. (9.9.2) and (9.7.1),

$$
e \cdot a_{4}^{L} \in H_{c_{4}} .
$$

Because $A_{4}=A_{4}^{T}+e \cdot a_{4}^{L}$, Lemma 9.8 follows from Eqs. (9.11) and (9.12).

The proof of Proposition 9.2 is completed by the observation that due to Lemma 9.8, $c_{4}=\left(A_{4}, \Phi_{4}\right)$ differs from $I(e)$ by an element of $H_{c_{4}}$.

Proof of Proposition 9.4. The configuration $c=(0, \theta) \in \mathfrak{C}_{0}$ is a solution to Eq. (5.4) if $\theta \in \mathfrak{s u}(2)$ is constant on $\mathbb{R}^{3}$. For this configuration, $\operatorname{ker} \mathfrak{D}_{c}=\operatorname{ker} \mathfrak{D}_{c}^{*}=\emptyset$. This follows from Eqs. (6.12) and (6.13). Hence the proposition is true when $n=0$.

For $n=1$, it is known that the Prasad-Sommerfield solution, $c_{1} \in \mathbb{C}_{1}$ [30] has $\operatorname{ker} \mathfrak{D}_{c}^{*} \cap H_{c}=0$ and $\operatorname{ker} \mathfrak{D}_{c} \cap H_{c}=\operatorname{ker} \mathfrak{D}_{c} \cap L_{2} \simeq \mathbb{R}^{4}$ [31]. The Prasad-Sommerfield solution is a solution to Eq. (5.4).

Proposition 9.4 follows from the next lemma. It is stated for $n>0$; the case $n<0$ can be obtained by reversing the orientation of $\mathbb{R}^{3}$.

Lemma 9.9. For each $n \geqq 2$, there exist configurations $c=c_{n} \in \mathbb{C}_{n}$ with $\operatorname{ker} \mathfrak{D}_{c}^{*} \cap H_{c}=\emptyset$, and $\operatorname{dim}\left(\operatorname{ker} \mathfrak{D}_{c} \cap L_{2}\right)=\operatorname{dim}\left(\operatorname{ker} \mathfrak{D}_{c} \cap H_{c}\right)=4 n$.

Proof of Lemma 9.9. In Chap 4 of [5], configurations $c\left(n, d, R,\left\{x_{\alpha}\right\}_{\alpha=1}^{n}\right) \in \mathfrak{C}_{n}$ were constructed to have the following properties:

(1) They are indexed by the data $\mathfrak{B}=\left\{d, R,\left\{x_{\alpha}\right\}_{\alpha=1}^{n}\right\}$, where $d>e^{4 R} \gg R>1$, and $\left\{x_{\alpha}\right\}_{\alpha=1}^{n} \subset \mathbb{R}^{3}$ satisfy $\inf _{\alpha \neq \beta}\left|x_{\alpha}-x_{\beta}\right|>d$.

(2) For each $\alpha \in(1, \ldots, n)$, there exists $g_{\alpha} \in C^{\infty}\left(\mathbb{R}^{3} ; G\right)$ such that $g_{\alpha} c(n, \mathfrak{B})$ $=c_{1}\left(x-x_{\alpha}\right)$ if $\left|x-x_{\alpha}\right|<R$. Here $c_{1}=\left(A_{1}, \Phi_{1}\right) \in \mathbb{C}_{1}$ is the unique [up to $C^{\infty}\left(\mathbb{R}^{4} \times G\right)$ ] Prasad-Sommerfield solution for which $\Phi_{1}(0)=0$.

(3) There exists $z<\infty$ which is independent of $n$ and the parameter $\mathfrak{B}$ with the following property:

Let $c(n, \mathfrak{B})=(A, \Phi)$. Then ||$\Phi|-1|(x) \leqq z \cdot \sum_{\alpha}\left(\left|x-x_{\alpha}\right|+1\right)^{-1}$, and $\left|* F_{A}+D_{A} \Phi\right|(x)$ $\leqq z \cdot \sum_{\alpha}\left(\left|x-x_{\alpha}\right|+1\right)^{-2}$.

(4) If $x \in \mathbb{R}^{3}$ satisfies $\inf _{\alpha}\left|x-x_{\alpha}\right|<R$, then $* F_{A}-D_{A} \Phi=0$. If $x \in \mathbb{R}^{3}$ satisfies $\inf _{\alpha}\left|x-x_{\alpha}\right|>R$, then $\left|* F_{A}-D_{A} \Phi\right|(x) \leqq z e^{-R} \cdot \sum_{\alpha}\left|x-x_{\alpha}\right|^{-2}$.

The first important property of $c(n, \mathfrak{B})$ is

Lemma 9.10. Let $c=c(n, \mathfrak{B}) \in \mathfrak{C}_{n}$ be as described above. There exists $R_{0}<\infty$ such that for $R>R_{0}, \operatorname{ker}\left(\mathfrak{D}_{c}^{*} \cap H_{c}\right)=\emptyset$. In this case, there exists $\gamma>0$ which is independent 
of the parameters $\mathfrak{B}$ such that for all $\psi \in H_{c}$,

$$
\left\|\mathfrak{D}_{c}^{*} \psi\right\|_{2} \geqq \gamma\|\psi\|_{H_{c}} .
$$

Proof of Lemma 9.10. This follows readily from Eqs. (6.12) and (6.13) and Property (4) above. To obtain the last statement of the lemma, one uses Lemma 7.6.

Now let $c_{1} \in \mathbb{C}_{1}$ and $\left\{g_{\alpha}\right\} \subset C^{\infty}\left(\mathbb{R}^{3} ; \mathrm{SU}(2)\right)$ be as in Property (2) above. Let $\left\{\psi_{i}\right\}_{i=1}^{4}$ be an $L_{2}$-orthonormal basis for $\operatorname{ker}\left(\mathfrak{D}_{c_{1}} \cap L_{2}\right)$. Define for each $\alpha \in(1, \ldots, n)$ and $i \in(1, \ldots, 4)$

$$
\psi_{\alpha i}=g_{\alpha}\left(\beta_{1 / 2 R}\left(x-x_{\alpha}\right) \psi_{i}\left(x-x_{\alpha}\right)\right) .
$$

Lemma 9.11. Let $c=c(n, \mathfrak{B}) \in \mathfrak{C}_{n}$ and let $\left\{\psi_{\alpha i}\right\}$ be as defined in Eq. (9.15). Let $\Pi\left\{\psi_{\alpha i}\right\}$ denote the $L_{2}$-orthogonal projection of $\left\{\psi_{\alpha i}\right\}$ onto $\operatorname{ker} \mathfrak{D}_{c}$. There exists $R_{0}<\infty$ such that for all $R>R_{0}$, $\operatorname{dim} \operatorname{Span} \Pi\left\{\psi_{\alpha i}\right\}=4 n$.

Proof of Lemma 9.11. Observe first that Property (1) implies that $\left\langle\psi_{\alpha i}, \psi_{\beta j}\right\rangle_{2}=0$ for $\alpha \neq \beta$ and for all $i, j$. For $\alpha=\beta$ one has

$$
\left\langle\psi_{\alpha i}, \psi_{\alpha j}\right\rangle_{2}-\delta_{i j} \mid \leqq z \cdot \sup _{i}\left\|\left(1-\beta_{R / 2}\right) \psi_{i}\right\|_{2}
$$

where $z<\infty$ is independent of the parameters $\mathfrak{B}$. For $R$ sufficiently large, Eq. (9.13) implies that $\operatorname{dim} \operatorname{Span}\left\{\psi_{\alpha i}\right\}=4 n$. Now, observe that

$$
\Pi \psi_{\alpha i}=\psi_{\alpha i}+\mathfrak{D}_{c}^{*} v_{\alpha i},
$$

where $v_{\alpha i} \in H_{c}\left(\hat{\mathrm{g}}^{\prime}\right)$ is the unique solution to

$$
\mathfrak{D}_{c} \mathfrak{D}_{c}^{*} v_{\alpha i}+\sum_{i=1}^{3}\left(\frac{\partial \beta_{1 / 2 R}}{\partial x_{j}}\left(x-x_{\alpha}\right) \tau^{j}\right) g_{\alpha} \psi_{i}\left(x-x_{\alpha}\right)=0 \text {. }
$$

Equation (9.15) follows from Property (2) and the definition of $\left\{\psi_{i}\right\}$. Using Eq. (9.15), and then Hölder's inequality with Lemma 9.11, one obtains

$$
\begin{aligned}
\left\|\mathfrak{D}_{c}^{*} v_{\alpha i}\right\|_{2}^{2} & \leqq \text { const }\left\langle\left|d \beta_{R / 2}\left(x-x_{\alpha}\right)\right|\left|v_{\alpha i}\right|,\left|\psi_{i}\left(x-x_{\alpha}\right)\right|\right\rangle_{2} \\
& \leqq \text { const } \sup _{i}\left\|\left(1-\beta_{1 / 4 R}\right) \psi_{i}\right\|_{2}^{2} .
\end{aligned}
$$

The second line above uses Lemma 6.3. Lemma 9.11 follows directly from Eqs. (9.13)-(9.16).

Lemma 9.9 is a direct corollary to Lemmas 9.10 and 9.11 and the next result

Lemma 9.12. Let $c=c(n, \mathfrak{B}) \in \mathfrak{C}_{n}$. There exists $R_{0}<\infty$ such that for all $R>R_{0}$, $\operatorname{dim}\left(\operatorname{ker} \mathfrak{D}_{c} \cap H_{c}\right)=4 n$.

Proof of Lemma 9.12. Assume that the lemma is false and a contradiction exists. Indeed, if the lemma were not true, then for some $n$, and for every set of parameters $(n, \mathfrak{B})$, there exists $\psi=\psi(\mathfrak{B}) \in \operatorname{dim}\left(\operatorname{ker} \mathfrak{D}_{c(n, \mathfrak{B})} \cap H_{c}\right)$ which is $H_{c}$-orthogonal to $\left\{\Pi \psi_{\alpha i}\right\}$, and also satisfies $\|\psi\|_{c}=1$. Equations (6.6), (6.12), (6.13), and Lemma 6.8 in conjunction with Property (3) imply the following: There exist constants $0<\mu$, $r<\infty$ which are independent of the parameters $\mathfrak{B}$ such that for some $\alpha \in(1, \ldots, n)$

$$
\int_{\left|x-x_{\alpha}\right|<r} d^{3} x|\psi|^{2} \geqq \mu \text {. }
$$


The proof of Eq. (9.17) is similar to the proof of Lemma 6.6. Given $c(n, \mathfrak{B})$ and $\psi$ as above, choose $\alpha$ so that Eq. (9.17) holds. Let $c_{1}, g_{\alpha}$ be as specified in Property (2) and let

$$
\psi_{R}=\beta_{1 / 2 R}\left(g_{\alpha}^{-1} \psi\right)\left(x+x_{\alpha}\right)
$$

Observe that

$$
\mathfrak{D}_{c_{1}} \psi_{R}=\left(\sum_{j=1}^{3} \frac{\partial \beta_{1 / 2 R}}{\partial x_{j}} \tau^{j}\right) \cdot\left(g_{\alpha}^{-1} \psi\right)\left(x+x_{\alpha}\right),
$$

and for each $i \in(1, \ldots, 4)$,

$$
\begin{aligned}
\left\langle\psi_{R}, \psi_{i}\right\rangle_{c_{1}} & =\left\langle\psi, \psi_{\alpha i}\right\rangle_{c} \\
& =-\left\langle\psi, \mathfrak{D}_{c}^{*} v_{\alpha i}\right\rangle_{c} .
\end{aligned}
$$

From Eqs. (9.20), (9.15), and (6.6) one obtains the a priori bound

$$
\left|\left\langle\psi_{R}, \psi_{i}\right\rangle_{c_{1}}\right| \leqq \text { const } \sup _{i}\left\|\left(1-\beta_{R / 4}\right) \psi_{i}\right\|_{2}
$$

Now consider the sequence $\left\{\psi_{R}\right\}$ as $R \rightarrow \infty$. This is a bounded sequence in $H_{c_{1}}$, so it has a weakly convergent subsequence in $H_{c_{1}}$. Due to Eq. (9.17) and the Rellich lemma, the limit is nontrivial. Due to Eq. (9.19), the limit is in ker $\mathscr{D}_{c_{1}}$. Due to Eq. (9.21), the limit is linearly independent of $\left\{\psi_{i}\right\}_{i=1}^{4}$. This contradicts the assumption that $\operatorname{dim}\left(\operatorname{ker} \mathscr{D}_{c_{1}} \cap H_{c_{1}}\right)=4$. Hence, Lemma 9.12 is true.

\section{References}

1. Atiyah, M.F.: Geometry of Yang-Mills fields (Fermi lectures). Scuola Normale Superiore, Pisa (1979)

2. Bourguignon, J.P., Lawson, H.B., Jr.: Yang-Mills theory; its physical origin and differential geometric aspects. In: Sminar on differential geometry, Yau, S.-T. (ed.). Ann. Math. Stud. 102, Princeton (1982)

3. Bourguignon, J.P., Lawson, H.P., Jr., Simons, J.: Stability and gap phenomena for Yang-Mills fields. Proc. Natl. Acad. Sci. (USA) 76, 1550 (1979)

4. Atiyah, M.F., Jones, J.D.S.: Topological aspects of Yang-Mills theory. Commun. Math. Phys. 61, 97 (1978)

5. Jaffe, A., Taubes, C.H.: Vortices and monopoles. Boston: Birkhäuser 1980

6. Taubes, C.H.: The existence of a non-monimal solution to the SU(2) Yang-Mills-Higgs equations on $\mathbb{R}^{3}$, Part I: Commun. Math. Phys. 86, 257 (1982); Part II : Commun. Math. Phys. 86, 299 (1982)

7. Hitchin, N.: Compact, four dimensional Einstein manifolds. J. Diff. Geom. 9, 435 (1974) Yau, S.-T.: On the Ricci curvature of a compact Kähler manifold and the complex Monge Ampère equation. I. Commun. Pure Appl. Math. 31, 339 (1978)

8. Penrose, R.: Nonlinear gravitons and curved twistor theory. Gen. Rel. Grav. 7, 31 (1976) Gibbons, G.W., Pope, C.B.: The positive action conjecture and asymptotically Euclidean metrics in quantum gravity. Commun. Math. Phys. 61, 239 (1978)

Hitchin, N.: Polygons and gravitons. Proc. Camb. Philos. Soc. 85, 465 (1979)

9. Palais, R.: Ljusternik-Snirelman theory on Banach manifolds. Topology 5, 115 (1966)

10. Eells, J., Wood, J.C.: Harmonic maps from surfaces to complex projective spaces (preprint)

11. Segal, G.: Topology of spaces of rational functions. Acta Math. 143, 39 (1979)

12. Weinberg, E.: Parameter counting for multi-monopole solutions. Phys. Rev. D 20, 936 (1976)

13. Parker, T.: Gauge theories on 4-dimensional, Riemannian manifolds. Commun. Math. Phys. 85, $563(1982)$ 
14. Freed, D., Freedman, M., Uhlenbeck, K.K.: Gauge theories and 4-manifolds. MSRI Berkeley preprint (1983)

15. Bourguignon, J.P., Lawson, H.B., Jr.: Stability and isolation phenomena for Yang-Mills fields. Commun. Math. Phys. 79, 189 (1981)

16. DeTurck, D., Kazdan, J.L.: Some regularity theorems in Riemannian geometry. Ann. Sci. Ec. Norm. Sup. 14, 249 (1981)

17. Atiyah, M.F., Hitchin, N.J., Singer, I.M.: Self-duality in 4-dimensional Riemannian geometry. Proc. R. Soc. London A 362, 425 (1978)

18. Uhlenbeck, K.: Chern numbers of Sobolev connections. Commun. Math. Phys. (to appear)

19. Forgacs, P., Manton, N.: Space-time symmetries in gauge theories. Commun. Math. Phys. 72, 15 (1980)

20. Aubin, T.: Nonlinear analysis on manifolds. Monge-Ampère equations. Berlin, Heidelberg, New York: Springer 1982

21. Parker, T., Taubes, C.H.: On Witten's proof of the positive energy theorem. Commun. Math. Phys. 84, 224 (1982)

22. Gromov, M., Lawson, H.B., Jr.: Positive scalar curvature and the Dirac operator on complete Riemannian manifolds (preprint)

23. Agmon, S.: Elliptic boundary value problems. New York: Van Nostrand 1965

24. Lockhart, R.B., McOwen, R.C.: On elliptic systems in $\mathbb{R}^{n}$. Acta Math. (to appear)

25. Nirenberg, L., Walker, H.: Null spaces of elliptic partial differential operators on $\mathbb{R}^{n}$. J. Math. Anal. Appl. 42, 271 (1973)

26. Callias, C.: Axial anomalies and index theorems on open spaces. Commun. Math. Phys. 62, 213 (1978)

27. Kato, T.: Perturbation theory for linear operators. Berlin, Heidelberg, New York: Springer 1980

28. Uhlenbeck, K.K.: Removable singularities in Yang-Mills fields. Commun. Math. Phys. 83, 11 (1982)

29. Ladyzhenskaya, O.A.: The mathematical theory of viscous incompressable flow. London: Gordon and Breach 1963

30. Prasad, M.K., Sommerfield, C.: Exact classical solution for the 't Hooft monopole and the JuliaZee dyon. Phys. Rev. Lett. 35, 760 (1975)

31. Maison, D.: Uniqueness of the Prasad-Sommerfield monopole solution. Nucl. Phys. B 182, 114 (1981)

Communicated by A. Jaffe

Received May 16, 1983 
